INSTITUT NATIONAL DE RECHERCHE EN INFORMATIQUE ET EN AUTOMATIQUE

\title{
Bounded extremal and Cauchy-Laplace problems on the sphere and shell
}

Bilal Atfeh — Laurent Baratchart — Juliette Leblond — Jonathan R. Partington

$\mathbf{N}^{\circ} 6504$

Avril 2008

Thème NUM

apport

de recherche 



\title{
Bounded extremal and Cauchy-Laplace problems on the sphere and shell
}

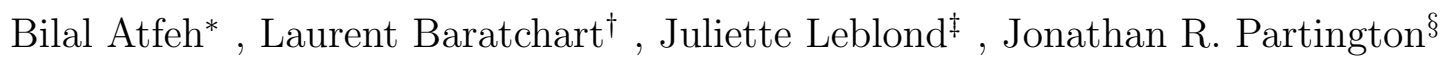 \\ Thème NUM - Systèmes numériques \\ Équipe-Projet Apics \\ Rapport de recherche $\mathrm{n}^{\circ} 6504-$ Avril 2008 -26 pages
}

\begin{abstract}
In this work, we develop a theory of approximating general vector fields on subsets of the sphere in $\mathbb{R}^{n}$ by harmonic gradients from the Hardy space $H^{p}$ of the ball, $1<p<\infty$. This theory is constructive for $p=2$, enabling us to solve approximate recovery problems for harmonic functions from incomplete boundary values. An application is given to Dirichlet-Neumann inverse problems for $n=3$, which are of practical importance in medical engineering. The method is illustrated by two numerical examples.
\end{abstract}

Key-words: Harmonic functions, Hardy classes, extremal problems, inverse Dirichlet-Neumann problems.

Math Subject Classifications: 31B05, 35J25, 42B35, 46E20, 47B35.

* INRIA, BP 93, 06902 Sophia-Antipolis Cedex, FRANCE

$\dagger$ INRIA, BP 93, 06902 Sophia-Antipolis Cedex, FRANCE, laurent.baratchart@sophia.inria.fr.

¥ INRIA, BP 93, 06902 Sophia-Antipolis Cedex, FRANCE, juliette.leblond@sophia.inria.fr.

$\S$ School of Mathematics, University of Leeds, Leeds LS2 9JT, U.K., j.r.partington@leeds.ac.uk.

Centre de recherche INRIA Sophia Antipolis - Méditerranée 2004, route des Lucioles, BP 93, 06902 Sophia Antipolis Cedex Téléphone : +334923877 77 -Télécopie : +33492387765 


\section{Problèmes extrémaux bornés et problèmes de Cauchy-Laplace dans des domaines sphèriques}

Résumé : Nous développons une théorie pour l'approximation de champs de vecteurs sur des sous-ensembles de la sphère dans $\mathbb{R}^{n}$ par des gradients harmoniques appartenant à l'espace de Hardy $H^{p}$ de la boule, $1<p<\infty$. Cette théorie est constructive pour $p=2$, et permet de résoudre des problèmes d'approximation de fonctions harmoniques à partir de valeurs incomplètes sur la frontière. Nous présentons une application aux problèmes inverses de Dirichlet-Neumann pour $n=3$, importante en ingéniérie médicale. La méthode est illustrée par deux exemples numériques.

Mots-clés : Fonctions harmoniques, classes de Hardy, problèmes extrémaux, problèmes inverses Dirichlet-Neumann. 


\section{Contents}

1 Introduction and notation 4

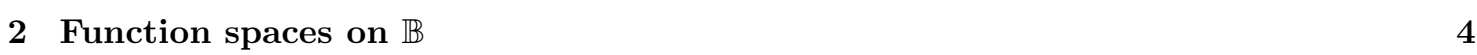

3 Bounded extremal problems

3.1 Approximation on the sphere . . . . . . . . . . . . . . . . . . 15

3.2 The case of the hemisphere in $\mathbb{R}^{3} \ldots \ldots \ldots \ldots \ldots \ldots \ldots$

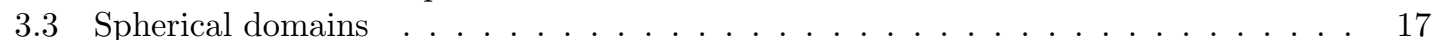

$\begin{array}{lll}4 & \text { Numerical computations } & 19\end{array}$

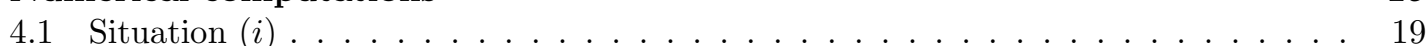

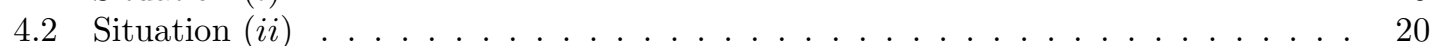

5 Conclusions 24 


\section{Introduction and notation}

Fix an integer $n \geq 2$ (in applications we shall usually require $n=3$ ). We write $\mathbb{B}$ for the open unit ball $\mathbb{B}=\left\{x \in \mathbb{R}^{n}:|x|<1\right\}$, and $\mathbb{S}$ for the sphere $\mathbb{S}=\left\{x \in \mathbb{R}^{n}:|x|=1\right\}$.

The techniques introduced in this paper will enable us to extend a standard approximation problem for holomorphic functions in one complex variable [2, 6, 11, 19] to the real multi-dimensional situation, enabling the approximate recovery of harmonic functions in $\mathbb{B}$ from incomplete and noisy data on a proper subset $K \subset \mathbb{S}$. We also consider the case of the shell $\mathbb{G}=\mathbb{B} \backslash \rho \overline{\mathbb{B}}$ $(0<\rho<1)$ bounded by two spheres; the same techniques allow us to recover harmonic functions from measurements on a proper subset $K \subset \partial \mathbb{G}$.

We proceed by finding the best quadratic approximant of a given vector field on $K \subset \partial \mathbb{G}$ among traces of gradients of harmonic functions in $\mathbb{G}$, which satisfy a norm constraint on the complementary subset $\partial \mathbb{G} \backslash K$ of the boundary.

This represents a multi-dimensional generalization of previous two-dimensional work cited above, where bounded extremal problems are considered in the Hardy spaces of the unit disk and annulus of the complex plane $\mathbb{C} \simeq \mathbb{R}^{2}$. There, analytic and harmonic functions are classically linked by the Cauchy-Riemann equations, while in $\mathbb{R}^{n}$, we define analytic functions to be gradients of harmonic functions, as in the work of Stein and Weiss [26, 27, 28. This also provides a generalization of the classical Hardy spaces to functions defined on a ball or a half-space in $\mathbb{R}^{n}$, and gives an appropriate setting for the approximation problem we consider.

A motivation for these problems comes from Cauchy-type issues for the Laplace operator, arising in non-destructive control. There, Dirichlet and Neumann data are both available on a subset, say $K$, of the boundary of a domain $\mathcal{D}$ in which the associated function is harmonic. This furnishes the trace $F$ on $K$ of a function which lies in a Hardy space of harmonic gradients in $\mathcal{D}$. The issue thus becomes that of recovering such a function from its trace on $K$. This is an ill-posed problem and furthermore, in practical situations, Dirichlet and Neumann data are provided by experimental measurements which are necessarily corrupted. As a consequence, the associated function $F$ is not, in general, exactly the trace on $K$ of a Hardy function. Thus, the above issue is to be seen as an approximation problem on $K$, and a constraint on the complementary part of the boundary (which plays the role of a regularization parameter) is required for the problem to be well-posed.

Among the many applications in which this problem arises, we mention the inverse EEG (electroencephalography) problem, from medical engineering [16, 17, 18]. There, the head is regarded as being made of several homogeneous spherical layers of different constant conductivities (the scalp, the skull, the brain) and the data are pointwise values of the electrical potential (measured by electrodes) and the current flux, both available on part of the scalp. The issue is to locate (dipolar) current sources sited in the brain, see e.g. [7, and may require a preliminary step, called "cortical mapping", of data translation from the scalp to the surface of the brain, modelled as a ball. This requires the solution of several Cauchy problems in shells, with incomplete data, which may be approached with the approximation techniques that we develop below.

The paper is organized as follows. Section 2 introduces function spaces $H^{p}$ of Hardy type on the $\mathbb{R}^{n}$ ball and establishes some of their fundamental properties. This is the basis for Section 3. in which the bounded extremal problems are presented and explicitly solved for $p=2$ and $n=3$. Finally, this method is illustrated in Section 4 by some numerical experiments, and some concluding remarks are made in Section 5 .

\section{Function spaces on $\mathbb{B}$}

For $1 \leq p<\infty$, by analogy with the classical theory (see [26, VII.3.2,VII.4.1]), one defines $H^{p}=H^{p}(\mathbb{B})$ to be the space of gradient vector fields $G=\nabla g$ for some $g$ harmonic in $\mathbb{B}$ such that

$$
\|G\|_{p}:=\left(\sup _{0 \leq r<1} \int_{\mathbb{S}}|G(r x)|^{p} d \sigma(x)\right)^{1 / p}<\infty
$$


where $\sigma$ denotes normalized surface area on $\mathbb{S}$. Moreover, $H^{\infty}=H^{\infty}(\mathbb{B})$ is the space of such fields $G$ that are uniformly bounded in $\mathbb{B}$, equipped with the supremum norm; we let $H^{c} \subset H^{\infty}$ be the closed subspace of continuous $G$ on $\bar{B}$.

Clearly we may, and we will, assume that $g(0)=0$. In this paper we are essentially concerned with the range $1<p<\infty$, but we include $H^{1}, H^{c}$ and $H^{\infty}$ in the general statements of this section where it would have been unnatural to omit them. Hopefully this lays ground for further study.

Gradients of harmonic functions are also referred to as Riesz systems; as in [3, 4, these may be decomposed into a radial component $\partial_{n} g$ along the outer normal and a tangential component $\nabla_{\mathbb{S}} g$, both defined on $\mathbb{S}$. Indeed, each component of $G$ is harmonic in $\mathbb{B}$ hence, since (1) holds, it follows from a classical theorem of Fatou that $G$ has non-tangential limits on $\mathbb{S}$ almost everywhere. These define a boundary function in $L^{p}\left(\mathbb{S} ; \mathbb{R}^{n}\right):=L^{p}\left(\mathbb{S}, \sigma ; \mathbb{R}^{n}\right)$, of which $G$ is the Poisson integral; when $p=1$ this last fact depends on a generalization of the $\mathrm{F}$. and M. Riesz theorem to harmonic gradients whose half-space version [26, VII.3.2, Thm 6] is easily carried over to the ball using Kelvin transforms [5, Ch. 7]. Moreover, if we let $G_{r}(x):=G(r x)$ and still denote the boundary function by $G$, then $\left\|G_{r}-G\right\|_{L^{p}\left(\mathbb{S} ; \mathbb{R}^{n}\right)}$ tends to 0 as $r$ tends to $1^{-}$for $1 \leq p<\infty$ ( $[5$, Ch. 6] or [26, VII.4.1]); see also [27, [28, VI.4] and [26, VII.3.1], where it is shown that $|G|^{p}$ is subharmonic, hence $\left\|G_{r}\right\|_{L^{p}\left(\mathbb{S} ; \mathbb{R}^{n}\right)}$ is an increasing function of $r \in(0,1)$ by the Green formula. If $G \in H^{\infty} \backslash H^{c}$, then $G_{r}$ need not converge in $L^{\infty}\left(\mathbb{S} ; \mathbb{R}^{n}\right)$, but it converges at least weak-* to $G$. The monotonicity of $\left\|G_{r}\right\|_{L^{\infty}\left(\mathbb{S} ; \mathbb{R}^{n}\right)}$ follows from the subharmonicity of $|G|$. Therefore in all cases $\|G\|_{L^{p}\left(\mathbb{S} ; \mathbb{R}^{n}\right)}=\|G\|_{p}$, as follows from Minkowski's inequality for integrals applied to the Poisson representation. Consequently $H^{p}$ can be regarded isometrically as a closed subspace of $L^{p}\left(\mathbb{S} ; \mathbb{R}^{n}\right)$ when $G$ is identified with its nontangential limit. Moreover, if $G \in H^{p}$ happens to lie in $L^{q}\left(\mathbb{S} ; \mathbb{R}^{n}\right)$ for some $q>p$, then $G \in H^{q}$.

Suppose that $G=\nabla g \in H^{p}$, where $g$ is harmonic in $\mathbb{B}$ and $g(0)=0$. We may define $g$ almost everywhere on $\mathbb{S}$ by taking radial limits; their existence follows by elementary integration from the existence of $\lim _{r \rightarrow 1} G(r x)$ for almost every $x \in \mathbb{S}$. For such $x$, we have by convexity when $1 \leq p<\infty$

$$
|g(x)|^{p} \leq\left(\int_{0}^{1}|G(r x)| d r\right)^{p} \leq \int_{0}^{1}|G(r x)|^{p} d r
$$

and since $\left\|G_{r}\right\|_{L^{p}\left(\mathbb{S} ; \mathbb{R}^{n}\right)}$ increases with $r$, we obtain upon integrating the above inequality against $\sigma$ that $\|g\|_{L^{p}(\mathbb{S})} \leq\|G\|_{p}$, letting $L^{p}(\mathbb{S}):=L^{p}(\mathbb{S} ; \mathbb{R})$. Applying this to $g-g_{r}$ gives us

$$
\left\|g-g_{r}\right\|_{L^{p}(\mathbb{S})} \leq r\left\|G-G_{r}\right\|_{L^{p}\left(\mathbb{S} ; \mathbb{R}^{n}\right)}
$$

hence $g_{r}$ converges to $g$ in $L^{p}(\mathbb{S})$ which entails that $g$ is the Poisson integral of its non-tangential limit. Moreover, Jensen's inequality easily implies that $|g|^{p}$ is subharmonic, therefore $\left\|g_{r}\right\|_{L^{p}(\mathbb{S})}$ is again an increasing function of $r \in(0,1)$. When $p=\infty$, it follows from the mean-value theorem that $\|g\|_{L^{\infty}(\mathbb{S})} \leq\|G\|_{\infty}$ and that $g_{r}$ tends to $g$ in $L^{\infty}(\mathbb{S})$, while the monotonicity of $\left\|g_{r}\right\|_{L^{\infty}(\mathbb{S})}$ is obvious from the maximum principle.

Observe that if $n=2$ and $\mathbb{R}^{2}$ is identified with $\mathbb{C}$ under the map $(x, y) \mapsto x-i y$, then the Hardy spaces we just defined coincide with the standard Hardy spaces of the disk while $H^{c}$ is the so-called disk algebra.

By means of the reflection $R(X)=X /|X|^{2}$, we may define the Kelvin transform $\mathcal{K}[h]$ of a function $h$ defined on a set $\Omega \subset \mathbb{R}^{n}$ by

$$
\mathcal{K}[h](X)=\frac{1}{|X|^{n-2}} h(R(X)) \quad \text { for } \quad X \in R(\Omega) .
$$

When $\Omega$ is open, it turns out that $h$ is harmonic in $\Omega$ if, and only if $\mathcal{K}[h]$ is harmonic in $R(\Omega)(\mathrm{cf}$. [5. Thm 4.7]). Note that the Kelvin transform is an involution, that is, $\mathcal{K}[\mathcal{K}[h]]=h$. When $\Omega$ contains the complement of a ball, $h$ is said to be harmonic at infinity if $\mathcal{K}[h]$ is harmonic at 0 .

With this convention, we define analogously $H_{-}^{p}=H^{p}\left(\mathbb{B}^{e}\right)$, where $\mathbb{B}^{e}=\{\infty\} \cup\left(\mathbb{R}^{n} \backslash \overline{\mathbb{B}}\right)$, consisting of gradients $G=\nabla g$ of harmonic functions in $\mathbb{B}^{e}$ that satisfy (11) (resp. are bounded if $p=\infty$ ), 
where this time $1<r<\infty$. We again normalize $g$ so that $g(\infty)=0$, although this is automatically the case if $n \geq 3\left[\underline{5}\right.$, Thm 4.8]. The space $H_{-}^{c}$ consists of $H_{-}^{\infty}$-functions that are continuous for $|X| \geq 1$.

In view of (2) and the formula

$$
\nabla \mathcal{K}[h](X)=\frac{(\nabla h)(R(X))}{|X|^{n}}-2^{t} X \cdot(\nabla h)(R(X)) \frac{X}{|X|^{n+2}}-(n-2) h(R(X)) \frac{X}{|X|^{n}},
$$

where the left superscript " $t$ " indicates the transpose and the dot "." the Euclidean scalar product, it is readily seen, when $g$ is harmonic in $\mathbb{B}$ with $g(0)=0$, that $\nabla g \in H^{p}$ if and only if $\nabla \mathcal{K}[g] \in H_{-}^{p}$. Moreover, from (3), we deduce if $G \in H_{-}^{p}$ that $|G(X)|=O\left(1 /|X|^{n}\right)$. Using the Green formula and the subharmonicity of $|G|^{p}$, we then conclude that $\left\|G_{r}\right\|_{L^{p}\left(\mathbb{S} ; \mathbb{R}^{n}\right)}$ decreases with $r$ on $[1,+\infty)$. In particular, we again have that $\|G\|_{p}=\|G\|_{L^{p}\left(\mathbb{S} ; \mathbb{R}^{n}\right)}$. Notice, if we write $G=\nabla g$, that $\|G\|_{p}$ and $\|\nabla \mathcal{K}[g]\|_{p}$ are equivalent but not equal when $n>2$. More precisely, we certainly have $\nabla_{\mathbb{S}} g=$ $\nabla_{\mathbb{S}} \mathcal{K}[g]$ since $g$ and $\mathcal{K}[g]$ coincide on $\mathbb{S}$, but taking the scalar product in (3) against the normal vector $x$ at $x \in \mathbb{S}$, we have

$$
\partial_{n} \mathcal{K}[g](x)=-\partial_{n} g(x)-(n-2) g(x) .
$$

The natural embedding of $\mathbb{S}$ into $\mathbb{R}^{n}$ makes the sphere a Riemannian manifold, the scalar product between tangent vectors at a point being just their scalar product in $\mathbb{R}^{n}$. This allows one to define the gradient $\nabla_{\mathbb{S}} \Phi(x) \in \mathbb{R}^{n}$ at $x \in \mathbb{S}$ of a function $\Phi: \mathbb{S} \rightarrow \mathbb{R}$ which is differentiable at $x$, meaning it is differentiable there in some (hence any) smooth system of charts. Clearly $\nabla_{\mathbb{S}} \Phi(x)$ is tangent to $\mathbb{S}$ at $x$, and coincides with the usual gradient at $x$ of the radial extension $\Phi(X /|X|)$ of $\Phi$ to $\mathbb{R}^{n} \backslash\{0\}$.

For $1 \leq p<\infty$ we define the Sobolev space $W^{1, p}(\mathbb{S})$ to be the space of all functions $f$ such that, if $\left(U_{j}, \psi_{j}\right)_{1 \leq j \leq N}$ is a smooth system of charts that covers $\mathbb{S}$ and $\left(\varphi_{j}\right)$ a smooth partition of unity subordinated to $\left(U_{j}\right)$, then $\left(f \varphi_{j}\right) \circ \psi_{j}^{-1}$ lies in the standard Sobolev space $W^{1, p}\left(\psi_{j}\left(U_{j}\right)\right)$ for all $j$. The latter consists of those functions in $L^{p}\left(\psi_{j}\left(U_{j}\right)\right)$ for which the first-order partial derivatives exist (in the weak sense) and define functions in $L^{p}\left(\psi_{j}\left(U_{j}\right)\right)$. It is easily checked that the definition does not depend on the finite system of smooth charts that is chosen (see [21, Ch. 1]). Using [26. V.2, Prop.1], it is straightforward that $f \in W^{1, p}(\mathbb{S})$ if and only if there is sequence of smooth functions on $\mathbb{S}$ that converges to $f$ in $L^{p}(\mathbb{S})$ and whose gradient vector field also converges in $L^{p}\left(\mathbb{S} ; \mathbb{R}^{n}\right)$. The latter limit, say $L$, does not depend on the sequence of smooth functions with the above properties, for ${ }^{t}\left(\varphi_{j} L+f \nabla_{\mathbb{S}} \varphi_{j}\right) \circ \psi_{j}^{-1} D\left(\psi_{j}^{-1}\right)$ defines the distributional derivatives of $f \varphi_{j} \circ \psi_{j}^{-1}$ in $\psi_{j}\left(U_{j}\right)$. Thus $L$ can be taken as definition of $\nabla_{\mathbb{S}} f$. An intrinsic norm on $W^{1, p}(\mathbb{S})$ is then given by an expression such as

$$
\|f\|_{W^{1, p}(\mathbb{S})}^{p}=\|f\|_{L^{p}(\mathbb{S})}^{p}+\left\|\nabla_{\mathbb{S}} f\right\|_{L^{p}\left(\mathbb{S} ; \mathbb{R}^{n}\right)}^{p},
$$

for instance. We record for later use that, since $\left(f \varphi_{j}\right) \circ \Psi_{j}^{-1}$ is supported on a fixed compact set (namely supp $\varphi_{j} \circ \Psi_{j}^{-1}$ ), it follows from the Rellich-Kondrachov theorem [32, Thm 2.5.1] that $W^{1, p}(\mathbb{S})$ is compactly embedded into $L^{p}(\mathbb{S})$.

If $G=\nabla g \in H^{p}$ with $1 \leq p$, we saw when $r \rightarrow 1^{-}$that $g(r x)$ and $G(r x)$ converge in $L^{p}(\mathbb{S})$ and $L^{p}\left(\mathbb{S} ; \mathbb{R}^{n}\right)$ respectively to $g(x)$ and $G(x)$, therefore $g \in W^{1, p}(\mathbb{S})$. If $p=\infty$ then $G(r x)$ converges only weak-* to $G$ in $L^{\infty}\left(\mathbb{S} ; \mathbb{R}^{n}\right)$, but $g \in W^{1, p}(\mathbb{S})$ for all $1 \leq p<\infty$ and, by weak-* convergence, $\nabla_{\mathbb{S}} g$ is the tangential component of $G$ hence $g \in W^{1, \infty}(\mathbb{S})$.

In order to reconstruct functions defined on $\mathbb{S}$ from their values on proper subsets of $\mathbb{S}$, we shall require the following uniqueness result.

Lemma 1 Let $G \in H^{p}$ for some $1 \leq p \leq \infty$, and let $U$ be a nonempty relatively open subset of S. If $G_{\left.\right|_{U}}=0$ then $G \equiv 0$.

Proof: Without loss of generality we may take $p=1$ and $U$ to be connected. Since $G$ is given by the Poisson integral of a function that vanishes on $U$, it extends continuously to $\mathbb{B} \cup U$. Write $G=\nabla g$ and note that $g$ extends continuously to $\mathbb{B} \cup U$ and is constant on $U$. Subtracting this 
constant from $g$, and still denoting the resulting function by $g$, we may assume that $g_{\left.\right|_{U}}=0$. By means of the Kelvin transform $\mathcal{K}[g]$ which is harmonic in $\mathbb{B}^{e}$, we obtain a harmonic extension of $g$ to $\mathbb{B} \cup \mathbb{B}^{e} \cup U$ by defining $g(X)=-\mathcal{K}[g](X)$ for $X \in \mathbb{B}^{e}$; similarly there is an extension of $G=\nabla g$.

We can complete the proof by invoking Holmgren's uniqueness theorem [9. p. 47]; alternatively, we note that $g$ is real-analytic on its domain of definition, and all its tangential derivatives vanish on $U$. However, all partial derivatives of $g$ at any order are again harmonic and if $g$ is not identically zero on a neighbourhood of $U$, then one such derivative around some point of $U$ involves a function of the form $A(r-1)^{2}+O\left((r-1)^{3}\right)$, with $A \neq 0$, where we have set $r:=|X|$ for simplicity. This is a contradiction to the harmonicity for it prevents the mean-value property from holding (cf. 15 Cor. II.11]).

Hence $g$ vanishes on a nonempty open subset of its domain of definition, and it follows that it is identically zero.

Remark 1 Lemma 1 cannot be generalized to arbitrary subsets of positive measure, since in contrast to the well-known situation in two dimensions, an arbitrary subset of $\mathbb{S}$ with positive measure need not be a set of uniqueness for $H^{p}$ when $n \geq 3$. That is, there is a nonconstant harmonic function $g$ on $\mathbb{B}$, which can be taken to be $C^{1+\varepsilon}$ up to the boundary for some small $\varepsilon>0$, for which $G=\nabla g$ vanishes on a subset of $\mathbb{S}$ of positive measure. We refer to the papers of Bourgain, Wolff and Wang [10, 30, 31] for further details.

Let $\mathcal{H}_{k}$ be the space of homogeneous harmonic polynomials of degree $k$, and $\mathcal{H}_{\left.k\right|_{\mathrm{s}}}$ be the space of so-called spherical harmonics [5] Ch. 5, 10]. We further denote by $C(\mathbb{S})$ the space of continuous functions on $\mathbb{S}$.

Lemma 2 The space $\oplus_{k \geq 0} \mathcal{H}_{\left.k\right|_{\mathbb{S}}}$ of finite sums of spherical harmonics is dense in $L^{p}(\mathbb{S}), 1 \leq p<$ $\infty$, and it is uniformly dense in $C(\mathbb{S})$.

Proof: Every polynomial coincides on $\mathbb{S}$ with a sum of spherical harmonics [5, Cor. 5.7]. By the Stone-Weierstrass theorem, sums of spherical harmonics are therefore dense in $C(\mathbb{S})$, whence also in $L^{p}(\mathbb{S}), 1 \leq p<\infty$, cf. the proof of [5] Thm 5.8].

Below, we denote by $\sigma_{n-1}$ the unnormalized surface area of $\mathbb{S}$.

Lemma 3 For $p_{m} \in \mathcal{H}_{m}$, we have that

$$
\left(\sigma_{n-1} m(2 m+n-2)\right)^{1 / 2}\left\|p_{m}\right\|_{L^{2}(\mathbb{S})}=\left\|\nabla p_{m}\right\|_{L^{2}\left(\mathbb{S} ; \mathbb{R}^{n}\right)} .
$$

Proof: pick $0<r<1$. Applying the divergence formula to the vector field $p_{m} \nabla p_{m}$ on the domain $\mathbb{B} \backslash r \mathbb{B}$, we get by the harmonicity of $p_{m}$

$$
\int_{\mathbb{S}} \partial_{n} p_{m}(x) p_{m}(x) d \sigma-\int_{\mathbb{S}} \partial_{n} p_{m}(r x) p_{m}(r x) r^{n-1} d \sigma=\sigma_{n-1}^{-1} \int_{\mathbb{B} \backslash r \mathbb{B}}\left|\nabla p_{m}(y)\right|^{2} d y .
$$

Using Euler's theorem for homogeneous functions, we see that $\partial_{n} p_{m}$ equals $m p_{m}$ on $\mathbb{S}$ and $(m / r) p_{m}$ on $r \mathbb{S}$. Therefore, by the homogeneity of $p_{m}$ and $\nabla p_{m}$, we obtain

$$
m\left(1-r^{2 m+n-2}\right) \int_{\mathbb{S}} p_{m}^{2}(x) d \sigma=\sigma_{n-1}^{-1}\left(\int_{\mathbb{S}}\left|\nabla p_{m}(x)\right|^{2} d \sigma\right)\left(\int_{r}^{1} \rho^{2 m+n-3}, d \rho\right)
$$

which leads to (5).

Lemma 4 The space $\mathcal{G}$ spanned by the gradients of the spherical harmonics is dense in $H^{p}$ for $1 \leq p<\infty$ (resp. in $\left.H^{c}\right)$; hence the space spanned by the gradients of Kelvin transforms of spherical harmonics is dense in $H_{-}^{p}$ (resp. in $H_{-}^{c}$ ). 
Proof: $H^{c}$ is dense in $H^{p}$ if $1 \leq p<\infty$, hence it is sufficient to prove the assertion on $H^{c}$. Moreover, every $G \in H^{c}$ is the uniform limit of $G_{r}$ as $r \rightarrow 1^{-}$, therefore it is enough to show that $G$ is the limit of a sequence of gradients of harmonic polynomials, uniformly on compact subsets of $\mathbb{B}$.

For $m \in \mathbb{N}$ and $\xi \in \mathbb{S}$, denote by $Z_{m}(., \xi) \in \mathcal{H}_{m}$ the so-called zonal harmonic of degree $m$ with pole $\xi$, that is, the reproducing kernel of $\mathcal{H}_{m} \subset L^{2}(\mathbb{S})$ at $\xi[5$. Ch. 5]. Put $G=\nabla g$ where $g(0)=0$. By [5] Cor. 5.34], the series expansion in spherical harmonics

$$
g(y)=\sum_{m=1}^{\infty} \int_{\mathbb{S}} g(\xi) Z_{m}(y, \xi) d \sigma(\xi):=\sum_{m=1}^{\infty} p_{m}(y)
$$

converges, locally uniformly with respect to $y \in \mathbb{B}$. Clearly, all we need is to show that the series of gradients

$$
\sum_{m=1}^{\infty} \int_{\mathbb{S}} g(\xi) \nabla Z_{m}(y, \xi) d \sigma(\xi):=\sum_{m=1}^{\infty} \nabla p_{m}(y)
$$

also converges. Now, for fixed $\xi \in \mathbb{S}$, we get by homogeneity upon setting $x=y /|y|$ that

$$
\nabla Z_{m}(y, \xi)=|y|^{m-1} \nabla Z_{m}(x, \xi)=|y|^{m-1} \int_{\mathbb{S}} \nabla Z_{m}(\zeta, \xi) Z_{m}(\zeta, x) d \sigma(\zeta),
$$

where we used the reproducing property of the zonal harmonic. From Lemma 3 and the CauchySchwarz inequality, we thus get

$$
\left|\nabla Z_{m}(y, \xi)\right| \leq|y|^{m-1}\left(\sigma_{n-1} m(2 m+n-2)\right)^{1 / 2}\left\|Z_{m}(., \xi)\right\|_{L^{2}(\mathbb{S})}\left\|Z_{m}(., x)\right\|_{L^{2}(\mathbb{S})} .
$$

But

$$
\left\|Z_{m}(., \xi)\right\|_{L^{2}(\mathbb{S})}^{2}=\left\|Z_{m}(., x)\right\|_{L^{2}(\mathbb{S})}^{2}=Z_{m}(x, x) \leq C m^{n-2}
$$

for some constant $C$ by [5. Thm 5.27, Ex. 5.10], so that

$$
\left|\int_{\mathbb{S}} g(\xi) \nabla Z_{m}(y, \xi) d \sigma(\xi)\right| \leq C|y|^{m-1}\left(\sigma_{n-1} m(2 m+n-2)\right)^{1 / 2} m^{n-2}
$$

implying that (66) converges locally uniformly in $y \in \mathbb{B}$, as desired.

Remark 2 It is easy to see that Lemma 4 cannot hold for $p=\infty$, as $H^{\infty}$ contains discontinuous functions.

To state our next result, we introduce the canonical dual pairing between $L^{p}\left(\mathbb{S} ; \mathbb{R}^{k}\right)$ and $L^{q}\left(\mathbb{S} ; \mathbb{R}^{k}\right)$, where $1 / p+1 / q=1$ and $k \geq 1$ is an integer, which is given by

$$
\langle f, g\rangle=\int_{\mathbb{S}} f \cdot g d \sigma .
$$

Although this notation does not keep track of $k$, no confusion will arise.

Proposition 1 For $1 \leq p<\infty$ and $1 / p+1 / q=1, H^{p}$ is orthogonal to $H_{-}^{q}$ under the canonical pairing (7).

Proof: Again we may restrict ourselves to $p>1$. By Lemma 4 it is enough to show that, for any pair of non-negative integers $m, k$, one has $\left\langle\nabla p_{m}, \nabla \mathcal{K}\left[q_{k}\right]\right\rangle=0$ as soon as $p_{m} \in \mathcal{H}_{m}$ and $q_{k} \in \mathcal{H}_{k}$. Note that

$$
\mathcal{K}\left[q_{k}\right](X)=\frac{q_{k}(X)}{|X|^{2 k+n-2}} .
$$


Now, bearing in mind that the integrals are taken over $\mathbb{S}$, we have

$$
\begin{aligned}
\left\langle\nabla p_{m}, \nabla \frac{q_{k}}{|X|^{2 k+n-2}}\right\rangle & =\left\langle\nabla p_{m}, \nabla q_{k}\right\rangle-(2 k+n-2)\left\langle\nabla p_{m}, q_{k} X\right\rangle \\
& =\left\langle\nabla p_{m}, \nabla q_{k}\right\rangle-m(2 k+n-2)\left\langle p_{m}, q_{k}\right\rangle
\end{aligned}
$$

using Euler's theorem. Clearly this quantity is zero if $m=0$, and if $m=k>0$ it also vanishes, by [5, Lem. 5.13]. Otherwise,

$$
\begin{gathered}
\left\langle\nabla p_{m}, \nabla \frac{q_{k}}{|X|^{2 k+n-2}}\right\rangle=\left\langle\nabla p_{m}, \nabla q_{k}\right\rangle+\left\langle\nabla p_{m}, q_{k} \nabla \frac{1}{|X|^{2 k+n-2}}\right\rangle \\
=-(2 k+n-2) \sum_{i=1}^{n} \int_{\mathbb{S}} \partial_{x_{i}} p_{m} q_{k} x_{i} d \sigma(x)=-m(2 k+n-2) \int_{\mathbb{S}} p_{m} q_{k} d \sigma(x)=0,
\end{gathered}
$$

using Euler's theorem and the orthogonality of homogeneous harmonic polynomials of different degrees [5. Prop. 5.9].

In order to work with functions decomposed into a sum of functions in the Hardy classes $H^{p}$ and $H_{-}^{p}$, we need introduce those vector fields in $L^{p}\left(\mathbb{S} ; \mathbb{R}^{n}\right)$ whose tangential component is a gradient. Formally we define, for $1 \leq p \leq \infty$,

$$
\begin{aligned}
\mathcal{L}_{\nabla}^{p}(\mathbb{S})= & \left\{F(x)=f_{0}(x) x+\nabla_{\mathbb{S}} \phi:\right. \\
& \left.f_{0} \in L^{p}(\mathbb{S}), \phi \in W^{1, p}(\mathbb{S}), \int_{\mathbb{S}} f_{0} d \sigma=\int_{\mathbb{S}} \phi d \sigma=0\right\},
\end{aligned}
$$

and we let $\mathcal{L}_{\nabla}^{c}(\mathbb{S})$ indicate the continuous elements of $\mathcal{L}_{\nabla}^{\infty}(\mathbb{S})$. We shall customarily write $F=$ $\left(f_{0}, \nabla_{\mathbb{S}} \Phi\right)$, to single out the radial component $f_{0}$ of $F$, and its tangential component $\nabla_{\mathbb{S}} \phi$. The norm is induced by $L^{p}\left(\mathbb{S} ; \mathbb{R}^{n}\right)$, e.g. if $1 \leq p<\infty$ then

$$
\|F\|_{\mathcal{L}_{\nabla}^{p}(\mathbb{S})}^{p}=\int_{\mathbb{S}}\left[\left|f_{0}(x)\right|^{2}+\left|\nabla_{\mathbb{S}} \phi(x)\right|^{2}\right]^{p / 2} d \sigma(x),
$$

whence if $p=2$ :

$$
\|F\|_{\mathcal{L}_{\nabla}^{2}(\mathbb{S})}^{2}=\left\|f_{0}\right\|_{L^{2}(\mathbb{S})}^{2}+\left\|\nabla_{\mathbb{S}} \phi\right\|_{L^{2}\left(\mathbb{S} ; \mathbb{R}^{n}\right)}^{2} \cdot
$$

Clearly $\mathcal{L}_{\nabla}^{p}(\mathbb{S})$ is a Banach subspace of $L^{p}\left(\mathbb{S} ; \mathbb{R}^{3}\right)$. Note that $H^{p}$ is isometrically included in $\mathcal{L}_{\nabla}^{p}(\mathbb{S})$. Indeed, all we have to check is that $\partial_{n} g$ has zero-mean when $G=\nabla g \in H^{p}$, and this follows from the divergence formula for smooth vector fields:

$$
\begin{aligned}
\int_{\mathbb{S}} \partial_{n} g d \sigma=\int_{\mathbb{S}} G(x) \cdot x d \sigma(x) & =\lim _{r \rightarrow 1} \int_{\mathbb{S}} G(r x) \cdot x d \sigma(x) \\
& =\lim _{r \rightarrow 1}\left(\frac{1}{r^{2}} \int_{r \mathbb{B}} \Delta g(y) d y\right)=0
\end{aligned}
$$

where the $L^{p}\left(\mathbb{S} ; \mathbb{R}^{n}\right)$ - weak-* if $p=\infty$ - convergence of $G_{r}$ to $G$ was used. Within $\mathcal{L}_{\nabla}^{p}(\mathbb{S})$, members of $H^{p}$ are characterized by the fact that the tangential component is a vectorial Riesz transform of the normal component, see 3, 4 for Riesz transforms on the sphere.

Likewise, from (4), we get that $H_{-}^{p}$ is isometrically included in $\mathcal{L}_{\nabla}^{p}(\mathbb{S})$.

We now have the following decomposition.

Theorem 1 For $1<p<\infty$, there is a topological direct sum

$$
\mathcal{L}_{\nabla}^{p}(\mathbb{S})=H^{p} \oplus H_{-}^{p}
$$

The sum is orthogonal if $p=2$. 
Proof: We just need to show that $\mathcal{L}_{\nabla}^{p}(\mathbb{S}) \subset H^{p}+H_{-}^{p}$ for this makes the canonical map $H^{p} \oplus H_{-}^{p} \rightarrow$ $\mathcal{L}_{\nabla}^{p}(\mathbb{S})$ surjective, and since it is injective by Proposition 1 (and orthogonal if $p=2$ ) we can apply the open mapping theorem.

Let $F \in \mathcal{L}_{\nabla}^{p}(\mathbb{S})$, say, $F=\left(f_{0}, \nabla_{\mathbb{S}} \phi\right)$. Let $v_{0}$ be the solution to the Dirichlet problem

$$
\Delta v_{0}=0 \text { in } \mathbb{B}, \quad v_{0 \mid \mathrm{s}}=\phi .
$$

We claim that $\nabla v_{0} \in H^{p}$. Note that

$$
v_{0}(Z)=\int_{\mathbb{S}} P(\Xi, Z) \phi(\Xi) d \sigma(\Xi)
$$

where $P(\Xi, Z)$ denotes the Poisson kernel for $\mathbb{B}$, which is a constant multiple of $\frac{1-|Z|^{2}}{|\Xi-Z|^{n}}$; Thanks to the symmetry of the kernel's denominator with respect to $\Xi, Z$, the tangential component of $\nabla v_{0}$ to the sphere of radius $|Z|$ at a point $Z$ is the Poisson integral of $\nabla_{\mathbb{S}} \phi$, which is bounded in $L^{p}\left(\mathbb{S} ; \mathbb{R}^{n}\right)$ by Minkowski's inequality for integrals. The radial component of $\nabla v_{0}$ is bounded in $L^{p}(\mathbb{S})$ norm by a constant multiple of the norm of $\nabla_{\mathbb{S}} \phi$ (see the remark after Corollary 3.3 of $[3$. and [4 Eq. (2.2)]). This proves the claim.

Now

$$
F-\nabla v_{0}=\left(f_{0}-\partial_{n} v_{0}, 0\right)=\left(g_{0}, 0\right),
$$

where $g_{0} \in L^{p}(\mathbb{S})$ has vanishing mean. Put $L_{0}^{p}(\mathbb{S})$ for the space of such functions, and if $g \in L_{0}^{p}(\mathbb{S})$ denote by $u_{g}$ the solution with vanishing mean on $\mathbb{S}$ to the Neumann problem:

$$
\Delta u_{g}=0 \text { in } \mathbb{B}, \quad \partial_{n} u_{g \mid \mathrm{s}}=g .
$$

The non-tangential maximal function of $\left|\nabla u_{g}\right|$ lies in $L^{p}(\mathbb{S})$ [29] XVII, Thm 2.9], thus $\nabla u_{g} \in H^{p}$ and in particular $u_{g} \in W^{1, p}(\mathbb{S})$. Since the embedding $W^{1, p}(\mathbb{S}) \rightarrow L^{p}(\mathbb{S})$ is compact, so is the operator $\mathcal{N}: L_{0}^{p}(\mathbb{S}) \rightarrow L_{0}^{p}(\mathbb{S})$ given by $\mathcal{N}(g)=u_{g}$.

We claim that $2 I+(n-2) \mathcal{N}$ is injective. If $n=2$ there is nothing to prove. Otherwise, if it is not injective, there is $g \in L_{0}^{p}(\mathbb{S}) \backslash\{0\}$ such that $(n-2) u_{g} / 2=-g=-\partial_{n} u_{g}$. Let us first prove that $u_{g} \in L^{\alpha}(\mathbb{S})$ for all $1 \leq \alpha<\infty$. If $p>n$, then $u_{g}$ is continuous, since it lies in $W^{1, p}(\mathbb{S})$, by the Sobolev embedding theorem [1 Thm 5.4] applied in a finite system of charts; if $p=n$, the same theorem tells us that $u_{g} \in L^{\alpha}(\mathbb{S})$ for $\alpha \in[1, \infty)$. But if $p<n$ this theorem shows that $u_{g} \in L^{n p /(n-p)}(\mathbb{S})$ and the same must hold for $g$. Thus the regularity of the Neumann problem implies that $u_{g} \in W^{1, n p /(n-p)}(\mathbb{S})$ and proceeding inductively we find after $k$ steps that either $u_{g}$ is continuous or in $L^{\alpha}(\mathbb{S})$ for all $1 \leq \alpha<\infty$, or else $u_{g} \in W^{1, n p /(n-k p)}(\mathbb{S})$. When $k$ is so large that $n p /(n-k p) \geq n$ we get what we want. Now, as $r \rightarrow 1^{-}$, we know $\left(u_{g}\right)_{r}$ converges to $g$ in $L^{\alpha}(\mathbb{S})$ by standard properties of Poisson integrals [5. Thm 6.7], and that $\partial_{n}\left(u_{g}\right)_{r}$ converges to $\partial_{n} g$ in $L^{p}(\mathbb{S})$ in a dominated manner (that is, dominated by the nontangential maximal function of $\nabla u_{g}$ which belongs to $\left.L^{p}(\mathbb{S})\right)$. If we fix $1 / \alpha+1 / p=1$, we deduce that $\left(u_{g}\right)_{r} \partial_{n}\left(u_{g}\right)_{r}$ converges to $g u_{g}$ in $L^{1}(\mathbb{S})$, hence applying the divergence formula to $\left(u_{g}\right)_{r} \nabla\left(u_{g}\right)_{r}$ and letting $r$ tend to $1^{-}$gives us

$$
-\frac{2}{(n-2)} \int_{\mathbb{S}} g^{2}(x) d x=\int_{\mathbb{S}} u_{g}(x) \partial_{n} u_{g}(x) d \sigma(x)=\int_{\mathbb{B}}\left|\nabla u_{g}(y)\right|^{2} d y,
$$

where the monotone convergence was used in the last term. This is a contradiction because the first term is strictly negative, which proves the claim.

By the Fredholm theory [29, XVII, Thm 2.3], the operator $2 I+(n-2) \mathcal{N}$ is invertible, therefore there is a $g \in L_{0}^{p}(\mathbb{S})$ such that

$$
2 g+(n-2) u_{g}=-g_{0} .
$$

Since $u_{g}-\mathcal{K}\left[u_{g}\right]$ vanishes on $\mathbb{S}$ it holds that $\nabla_{\mathbb{S}}\left(u_{g}-\mathcal{K}\left[u_{g}\right]\right)=0$, and then (4) shows that

$$
\left(g_{0}, 0\right)=\nabla u_{g}-\nabla \mathcal{K}[g] \in H^{p}+H_{-}^{p} .
$$


Remark 3 Already when $n=2$, Theorem 1 does not hold for $p=1$ nor $p=\infty$.

From Lemma 4 and Theorem [1, it follows that the space of vector fields of the form $\left(p, \nabla_{\mathbb{S}} q\right)$ with $p, q$ polynomials is dense in $\mathcal{L}_{\nabla}^{p}(\mathbb{S})$ for $1<p<\infty$. In fact, it is dense in $\mathcal{L}_{\nabla}^{c}(\mathbb{S})$. Indeed, every function $\phi$ with continuous gradient on $\mathbb{S}$ extends to a $C^{1}$-function $\widetilde{\phi}$ on $\mathbb{R}^{n}$. On the cube $\left\{\left(x_{1}, \ldots, x_{n}\right) ;\left|x_{j}\right| \leq 1\right\}$, the function $\widetilde{\Phi}$ can be approximated uniformly by some polynomial $q$ in such a way that $\nabla \widetilde{\phi}$ is uniformly close to $\nabla q$; see 22 Thms $6.7,6.8]$ and observe, upon differentiating under the integral, that convolving with Jackson kernels allows one to approximate a function and its derivatives in a single stroke. Since any $f_{0} \in C(\mathbb{S})$ can be approximated uniformly by a polynomial, say, $p$, every $F=\left(f_{0}, \nabla_{\mathbb{S}} \phi\right) \in \mathcal{L}_{\nabla}^{c}(\mathbb{S})$ can be approximated by some polynomial vector field $\left(p, \nabla_{\mathbb{S}} q\right)$.

Considering the density property we just mentioned, it is important from the constructive viewpoint to carry out the decomposition of Theorem 1 algorithmically for polynomial vectors. Since every polynomial can be effectively reduced to a sum of spherical harmonics on $\mathbb{S}[5$, Cor. 5.7], it is enough to decompose $F=\left(p_{m}, \nabla_{\mathbb{S}} q_{m+1}\right)$, where $p_{m} \in \mathcal{H}_{m}(\mathbb{S})$ and $q_{m+1} \in \mathcal{H}_{m+1}(\mathbb{S})$ with $m>0$. On subtracting $\nabla q_{m+1} \in H^{p}$ to $F$, we are left to decompose $\left(\varphi_{m}, 0\right) \in \mathcal{L}_{\nabla}^{p}(\mathbb{S})$, where $\varphi_{m} \in \mathcal{H}_{m}(\mathbb{S})$. In view of (4) and Euler's theorem for homogeneous functions, the decomposition we seek is simply

$$
\left(\varphi_{m}, 0\right)=\frac{1}{2 m+n-2}\left(\nabla \varphi_{m}-\nabla \mathcal{K}\left[\varphi_{m}\right]\right) .
$$

Remark 4 Since one has an orthogonal sum $L^{2}(\mathbb{S})=\oplus_{m=0}^{\infty} \mathcal{H}_{m}$ [5, Thm 5.12], every $F \in \mathcal{L}_{\nabla}^{2}(\mathbb{S})$ can be written as

$$
F=\left(\sum_{m=1}^{\infty} \varphi_{m}, 0\right)+\nabla_{\mathbb{S}} v_{0}
$$

where $\varphi_{m} \in \mathcal{H}_{m}$ and $\nabla v_{0} \in H^{2}$. From what precedes, we see that the orthogonal projection of $F$ onto $H^{2}$ is given by

$$
P_{H^{2}} F=\nabla v_{0}+\nabla \sum_{m=0}^{\infty} \frac{1}{2 m+1} \varphi_{m}
$$

while

$$
P_{H_{-}^{2}} F=-\nabla \sum_{m=0}^{\infty} \frac{1}{2 m+1} \mathcal{K}\left[\varphi_{m}\right]=-\nabla \sum_{m=0}^{\infty} \frac{\varphi_{m}(X)}{(2 m+1)|X|^{2 m+n-2}} .
$$

For each closed subset $K \subset \mathbb{S}$, we write $\mathcal{L}_{\nabla}^{p}(K)$ for the space of restrictions to $K$ of functions in $\mathcal{L}_{\nabla}^{p}(\mathbb{S})$. The norm is also defined by restriction, namely for $1 \leq p<\infty$

$$
\|F\|_{\mathcal{L}_{\nabla}^{p}(K)}^{p}=\int_{K}|F|^{p} d \sigma
$$

and $\|F\|_{\mathcal{L}_{\nabla}^{\infty}(K)}=\sup _{K}|F|$. The space $\mathcal{L}_{\nabla}^{c}(K)$ consists of those elements of $\mathcal{L}_{\nabla}^{\infty}(K)$ that are continuous on $K$.

We then have the following density theorem, which enables us to approximate data on $K$ by the restrictions of Hardy functions. In some sense, this result accounts for the well-known ill-posedness of the Cauchy problem for the Laplace equation.

Theorem 2 Suppose that $1 \leq p<\infty$. If $K$ is closed and $\sigma(\mathbb{S} \backslash K)>0$, then $H_{\left.\right|_{K}}^{p}$ is dense in $\mathcal{L}_{\nabla}^{p}(K)$ and $H_{\left.\right|_{K} ^{c}}^{c}$ is dense in $\mathcal{L}_{\nabla}^{c}(K)$.

Proof: by the discussion after Remark 3 that led us to (10), it is enough to show that any member of $H_{-}^{c}$ of the form $\nabla \frac{q(X)}{|X|^{2 k+n-2}}$ with $q \in \mathcal{H}_{k}$ can be uniformly approximated on $K$ by some member of $H_{\left.\right|_{K}}^{c}$.

We modify a standard proof of Runge's theorem, as can be found in 23, for example. Without loss of generality we may assume that ${ }^{t}(0, \ldots, 0,-1) \in \mathbb{S} \backslash K$. Let $N={ }^{t}(0, \ldots, 0,1)$ and let $V_{K}$ 
denote an open neighbourhood of $K$ in $\mathbb{R}^{n}$ such that ${ }^{t}(0, \ldots, 0, z) \notin \bar{V}_{K}$ for $z \leq 0$.

Let $S$ denote the set of all real numbers $a \leq 0$ such that for each $k=0,1,2, \ldots$, every function of the form $\nabla \frac{q(X-a N)}{|X-a N|^{2 k+n-2}}$, with $q \in \mathcal{H}_{k}$ for some $k \geq 0$, is the uniform limit on $\overline{V_{K}}$ of functions of the form $\nabla g$ with $g$ harmonic on some neighbourhood of $\overline{\mathbb{B} \cup V_{K}}$. Such $\nabla g$ a fortiori belong to $H^{c}$, hence it is sufficient to prove that $0 \in S$.

Clearly $a \in S$ for all $a<-1$, because $\nabla \frac{q(X-a N)}{|X-a N|^{2 k+n-2}}$ is harmonic in $X$ on a neighbourhood of $\overline{\mathbb{B} \cup V_{K}}$ for such $a$. Moreover to each $a \leq 0$, there is a neighbourhood of $\overline{V_{K}} \times\{a\}$ in $\mathbb{R}^{n+1}$ on which $\nabla \frac{q(X-b N)}{|X-b N|^{2 k+n-2}}$ is harmonic in $X$ and real analytic in $(X, b)$ for all $q \in \mathcal{H}_{k}$ and all $k$. This shows at once that $S$ is closed, by the uniform continuity of $\nabla \frac{q(X-b N)}{|X-b N|^{2 k+n-2}}$ on $\overline{V_{K}} \times[a-\varepsilon, a+\varepsilon]$ for small $\varepsilon$, and entails if $a \in S$ that

$$
\nabla \frac{q(X-b N)}{|X-b N|^{2 k+n-2}}=\sum_{j=0}^{\infty} \frac{(b-a)^{j}}{j !} \nabla\left(\frac{\partial^{j}}{\partial z^{j}} \frac{q(X-z N)}{|X-z N|^{2 k+n-2}}\right)_{\mid z=a}
$$

where the expansion converges uniformly in $X \in \overline{V_{K}}$ for sufficiently small $|b-a|$. Since each partial derivative of $\frac{q(X)}{|X|^{2 k+n-2}}$ is of the form $\frac{p(X)}{|X|^{2 m+n-2}}$ for some $m \geq k$ and some $p \in \mathcal{H}_{m}$ [5, Lem. 5.15], we find that all gradients in the right-hand side of (11) are uniformly approximable on $\overline{V_{K}}$ by functions of the form $\nabla g$ with $g$ harmonic on some neighbourhood of $\overline{\mathbb{B} \cup V_{K}}$, so we conclude on truncating this series that $b \in S$ for all $b$ sufficiently close to $a$, that is, $S$ is open in $(-\infty, 0])$.

It follows by a connectedness argument that $S=(-\infty, 0]$, in particular $0 \in S$.

Remark 5 Already when $n=2$, Theorem [2] does not hold for $p=\infty$ [8].

Similar Hardy spaces can also be defined on the shell, that is, on the region $\mathbb{G}=\mathbb{B} \backslash \rho \overline{\mathbb{B}}$, where $0<\rho<1$. The Hardy space $H^{p}(\mathbb{G})$, for $1 \leq p<\infty$, is the space of gradient vector fields $G=\nabla g$ where $g$ is harmonic on $\mathbb{G}$, and such that the quantity

$$
\|G\|_{p, \mathbb{G}}:=\left(\sup _{\rho<r<1} \int_{\mathbb{S}}|G(r y)|^{p} d \sigma(y)\right)^{1 / p}
$$

is finite. The space $H^{\infty}(\mathbb{G})$ consists of those bounded members of $H^{1}(\mathbb{G})$ endowed with the supremum norm, and $H^{c}(\mathbb{G})$ is the subspace of $H^{\infty}(\mathbb{G})$-vector fields that extend continuously to $\overline{\mathbb{G}}$. It is easy to check that $H^{p}(\mathbb{G})$ and $H^{c}(\mathbb{G})$ are Banach spaces.

Clearly, the direct sum $H^{p}(\mathbb{B})+H^{p}\left(\rho \mathbb{B}^{e}\right)$ is mapped continuously into $H^{p}(\mathbb{G})$ under the ordinary addition of functions. This map is in fact a homeomorphism:

Proposition 2 The pointwise sum on $\mathbb{G}$ naturally induces topological direct sums

$$
H^{p}(\mathbb{G})=H^{p}(\mathbb{B}) \oplus H^{p}\left(\rho \mathbb{B}^{e}\right), 1 \leq p \leq \infty, \quad H^{c}(\mathbb{G})=H^{c}(\mathbb{B}) \oplus H^{c}\left(\rho \mathbb{B}^{e}\right) .
$$

Proof: by the open mapping theorem, it is enough to show that every $G \in H^{p}(\mathbb{G})$ can be uniquely decomposed as $G=G_{1}+G_{2}$ with $G_{1} \in H^{p}\left(\rho \mathbb{B}^{e}\right)$ and $G_{2} \in H^{p}$; this will settle the case of $H^{c}(\mathbb{G})$ as well, for if $G$ is continuous on $\overline{\mathbb{G}}$ then $G_{1}$ (resp. $G_{2}$ ) must be continuous up to $\rho \mathbb{S}(\operatorname{resp}$. $\mathbb{S}$ ) since $G_{2}$ (resp. $G_{1}$ ) is smooth across the latter by construction.

The uniqueness of the decomposition is clear, because if $G_{1}+G_{2}=G_{1}^{\prime}+G_{2}^{\prime}$ then $\left(G_{1}-G_{1}^{\prime}\right)_{\left.\right|_{\mathbb{S}}}$ and $\left(G_{2}^{\prime}-G_{2}\right)_{\left.\right|_{\mathbb{S}}}$ lie in $H_{-}^{c}$ and $H^{p}$ respectively, and they coincide a.e. on $\mathbb{S}$ so they must be zero by Proposition [1]

It remains to show the existence of such a decomposition. Suppose first that $1<p<\infty$. Let $G=\nabla g \in H^{p}(\mathbb{G})$ and assume for a while that $g$ is harmonic in a neighbourhood of $\mathbb{S}$. By Theorem 11 we can write $G_{\left.\right|_{\mathbb{S}}}=G^{+}+G^{-}$where $G^{+}=\nabla g^{+} \in H^{p}(\mathbb{B})$ and $G^{-}=\nabla g^{-} \in H^{p}\left(\mathbb{B}^{e}\right)$ and $\left\|G^{+}\right\|_{p}$, $\left\|G^{-}\right\|_{p}$ are bounded by a constant times $\|G\|_{p, \mathbb{G}}$. Put $g_{1}=g-g^{+}$on $\mathbb{G}$, and observe that $g_{\left.\right|_{\mathbb{S}}}$ 
and $g^{-}$, when considered as members of $W^{1, p}(\mathbb{S})$, have the same gradient namely the tangential component of $G^{-}$. Adding a constant to $g$ if necessary, we can therefore assume that $g_{1}$ and $g^{-}$ agree on $\mathbb{S}$. Then, the concatenated function $\widetilde{g}$ which is $g_{1}$ on $\mathbb{G}$ and $g^{-}$on $\mathbb{B}^{e}$ lies in $W_{l o c}^{1, p}\left(\rho \mathbb{B}^{e}\right)$, since it is absolutely continuous along almost every radius in the vicinity of $\mathbb{S} 32,2$, Rem. 2.1.5]. We claim that $\widetilde{g}$ is harmonic. Indeed, let $\phi$ be a smooth function with compact support in $\rho \mathbb{B}^{e}$. Since $\nabla g_{1}(r x)$ tends in $L^{p}\left(\mathbb{S}, \mathbb{R}^{n}\right)$ to $G(x)-G^{+}(x)=G^{-}(x)$ as $r \rightarrow 1^{-}$, the harmonicity of $g_{1}$ and the divergence formula for smooth vector fields yield

$$
\begin{aligned}
\int_{\mathbb{S}} \phi(x) G^{-}(x) \cdot x d \sigma & =\lim _{r \rightarrow 1} \int_{\mathbb{S}} \phi(r x) \partial_{n} g_{1}(r x) d \sigma \\
& =\lim _{r \rightarrow 1} \int_{\mathbb{G}} \nabla \phi(r y) \cdot \nabla g_{1}(r y) d y=\int_{\mathbb{G}} \nabla \phi \cdot \nabla g_{1} .
\end{aligned}
$$

Reversing the orientation on $\mathbb{S}$, a similar computation in $\mathbb{B}^{e}$ gives us

$$
-\int_{\mathbb{S}} \phi G^{-} . x d \sigma=\int_{\mathbb{B}^{e}} \nabla \phi . \nabla g^{-} .
$$

Adding up we get $\int_{\rho \mathbb{B} e} \nabla \phi . \nabla \widetilde{g}=0$, which means that $\widetilde{g}$ is a harmonic distribution thus a harmonic function by Weyl's lemma. This proves the claim.

The claim implies that $G_{1}:=\nabla \widetilde{g}$ lies in $H^{p}\left(\rho \mathbb{B}^{e}\right)$ and that $\left\|G_{1}\right\|_{L^{p}\left(\rho \mathbb{S} ; \mathbb{R}^{n}\right)}$ is less than a constant times $\|G\|_{p, \mathbb{G}}$. Hence the decomposition $G=G_{1}+G^{+}$on $\mathbb{G}$ meets our requirements.

We now remove the assumption that $g$ is harmonic in a neighbourhood of $\mathbb{S}$. Let $G \in H^{p}(\mathbb{G})$. By what precedes, to each $\rho<r<1$ there exist $G_{1} \in H^{p}\left(\rho \mathbb{B}^{e}\right)$ and $G^{+, r} \in H^{p}(r \mathbb{B})$ such that $G=G_{1}+G^{+, r}$ on $r \mathbb{B} \backslash \rho \overline{\mathbb{B}}$; moreover $\left\|G^{+, r}\right\|_{L^{p}\left(r \mathbb{S} ; \mathbb{R}^{n}\right)} \leq C\|G\|_{p, \mathbb{G}}$ for some constant $C$. Applying inductively the uniqueness of the decomposition, we get that $G_{1}$ is independent of $r$ and that $G^{+, r}=G_{r S}^{+, r^{\prime}}$ for $r \geq r^{\prime}$. Pick an increasing sequence $r_{n} \rightarrow 1^{-}$, and let $G_{2}$ be a weak limit point of the bounded sequence $G_{n}(x):=G^{+, r_{n}}\left(r_{n} x\right)$ of $H^{p}$-functions. Using weak convergence in the Poisson representation, we deduce since $G^{+, r_{n}}(y)=G^{+, r_{m}}(y)$ for $m \geq n$ that

$$
G^{+, r_{n}}(y)=\lim _{m \rightarrow \infty} G_{m}\left(y / r_{m}\right)=G(y), \quad|y|<r_{n} .
$$

Therefore $G=G_{1}+G_{2}$ on $\mathbb{G}$ which is the desired decomposition when $1<p<\infty$

If $G \in H^{\infty}$, pick $1<p<\infty$ and write $G=G_{1}+G_{2}$ with $G_{1} \in H^{p}\left(\rho \mathbb{B}^{e}\right)$ and $G_{2} \in H^{p}$, as above. Clearly $G_{1}$ is bounded on $\mathbb{S}$, therefore $G_{2}$ is also bounded so that in fact $G_{2} \in H^{\infty}$. Likewise, $G_{1}$ belongs to $H^{\infty}\left(\rho \mathbb{B}^{e}\right)$.

Assume finally that $p=1$ and pick $p^{\prime}>1$. If we let $\rho<r_{1}<r_{2}<1$, by the first part of the proof, we can write $G=G^{-, r_{1}}+G^{+, r_{2}}$ on $r_{2} \mathbb{B} \backslash r_{1} \overline{\mathbb{B}}$, where $G^{-, r_{1}} \in H^{p^{\prime}}\left(r_{1} \mathbb{B}^{e}\right)$ and $G^{+, r_{2}} \in H^{p^{\prime}}\left(r_{2} \mathbb{B}\right)$. Letting $r_{2} \rightarrow 1^{-}$, we observe since $\|G\|_{L^{1}\left(r_{2} \mathbb{S}\right)}$ and $\left\|G^{-, r_{1}}\right\|_{L^{1}\left(r_{2} \mathbb{S}\right)}$ are bounded that $\left\|G^{+, r_{2}}\right\|_{L^{1}\left(r_{2} \mathbb{S}\right)}$ is also bounded. Arguing as before, using this time weak-* convergence in the Poisson representation, we deduce that $G=G^{-, r_{1}}+G_{2}$ where $G_{2}$ is the Poisson integral of a finite measure on $\mathbb{S}$. Thus $\left\|G_{2}\right\|_{1}$ is bounded by Minkowski's inequality for integrals, that is, $G_{2}$ lies in $H^{1}$. Letting now $r_{1} \rightarrow \rho^{+}$, a similar argument shows that $G=G_{1}+G_{2}$ where $G_{1} \in H^{1}\left(\rho \mathbb{B}^{e}\right)$. This achieves the proof.

Proposition 2 entails that each $G \in H(\mathbb{G})$ has non-tangential limits almost everywhere on $\partial \mathbb{G}=$ $\mathbb{S} \cup \rho \mathbb{S}$, thereby defining a function in $L^{p}\left(\partial \mathbb{G}, \sigma ; \mathbb{R}^{3}\right)$, where we normalize the Lebesgue measure so that each sphere has unit measure. Moreover, if we denote again the boundary function by $G$, we have that $G_{r}$ converges to $G$ in $L^{p}\left(\mathbb{S} ; \mathbb{R}^{3}\right)$ (resp. $L^{p}\left(\rho \mathbb{S} ; \mathbb{R}^{3}\right)$ ) as $r \rightarrow 1^{-}$(resp. $r \rightarrow 1^{+}$if $1 \leq p<\infty$; if $p=\infty$, we only get weak-* convergence. In all cases, we deduce that $G(x)$ is the 
integral of its boundary values against the Poisson kernel of $\mathbb{G}$ at $x \in \mathbb{G}$ (see [5. Ch. 10]):

$$
P_{\mathbb{G}}(x, \xi)= \begin{cases}\sum_{m=0}^{\infty} \frac{1-(\rho /|x|)^{2 m+n-2}}{1-\rho^{2 m+n-2}} Z_{m}(x, \xi) & (\xi \in \mathbb{S}), \\ \sum_{m=0}^{\infty}|x|^{-m}\left(\frac{\rho}{|x|}\right)^{m+n-2} \frac{1-|x|^{2 m+n-2}}{1-\rho^{2 m+n-2}} Z_{m}\left(x, \frac{\xi}{|\xi|}\right) & (\xi \in \rho \mathbb{S}),\end{cases}
$$

where we recall the notation $Z_{m}(x, \xi)$ for the zonal harmonic with pole $\xi$. This provides us with an equivalent norm on $H^{p}(\mathbb{G})$ :

Corollary 1 For $1 \leq p \leq \infty$, the norm $\|G\|_{p, b}:=\|G\|_{L^{p}\left(\rho \mathbb{S} ; \mathbb{R}^{3}\right)}+\|G\|_{L^{p}\left(\mathbb{S} ; \mathbb{R}^{3}\right)}$ is equivalent to $\|G\|_{p, \mathbb{G}}$ on $H^{p}(\mathbb{G})$.

Proof: since $P_{\mathbb{G}}(x,$.$) is positive with integral 2$ on $\partial \mathbb{G}$, it follows from the Poisson representation of $G$ and Minkowski's inequality for integrals that $\|G\|_{p, \mathbb{G}} \leq 2\|G\|_{p, b}$ for $G \in H^{p}(\mathbb{G})$. Conversely, Proposition 2 implies that the identity map $\left(H^{p},\|\cdot\|_{p . \mathbb{G}}\right) \rightarrow\left(H^{p},\|\cdot\|_{p, b}\right)$ is continuous.

\section{Bounded extremal problems}

Let $K$ be a closed subset of $\mathbb{S}$ with nonempty interior $\stackrel{\circ}{K}$ and Lipschitz boundary $\partial K$. Notice the Lipschitz character entails that $\sigma(\partial K)=0$. A bounded extension operator $W^{1, p}(\stackrel{\circ}{K}) \rightarrow W^{1, p}(\mathbb{S})$ is obtained from the standard extension operator on $\mathbb{R}^{n-1}$ (see [26, VI.3.1]) on using a partition of unity subordinated to a system of charts. The existence of such an operator implies easily that $\mathcal{L}_{\nabla}^{p}(K)$ is a Banach space for $1 \leq p \leq \infty$.

In the rest of the paper, we assume that $K$ is a closed subset of $\mathbb{S}$ meeting the above properties, and we customarily denote its complement by $J=\mathbb{S} \backslash K$. Note that

$$
\mathcal{L}_{\nabla}^{p}(\mathbb{S})=\mathcal{L}_{\nabla}^{p}(K)+{ }_{\partial K} \mathcal{L}_{\nabla}^{p}(J)
$$

where $+_{\partial K}$ indicates that the sum is "fibred" over those pairs $\left(F_{K}, F_{J}\right) \in \mathcal{L}_{\nabla}^{p}(K) \times \mathcal{L}_{\nabla}^{p}(J)$ whose tangential components "agree" on $\partial K$. More precisely, there should exist $\phi_{K}, \phi_{J} \in W^{1, p}(\mathbb{S})$ and $f_{K}, f_{J} \in L^{p}(\mathbb{S})$ such that $F_{K}=\left(f_{K}, \nabla_{\mathbb{S}} \phi_{K}\right)_{\left.\right|_{K}}, F_{J}=\left(f_{J}, \nabla_{\mathbb{S}} \phi_{J}\right)_{\left.\right|_{J}}$, and $\left(\phi_{K}\right)_{\left.\right|_{\partial K}}=\left(\phi_{J}\right)_{\left.\right|_{\partial K}}$ where the trace on the boundary of a Lipschitz domain is defined as in Sobolev spaces on $\mathbb{R}^{n}$ using a system of charts [32 Rem. 4.4.5]. The coincidence of the traces on $\partial K$ is equivalent to the belonging of the concatenated function $\left(\phi_{K}\right)_{\left.\right|_{K}} \vee\left(\phi_{J}\right)_{\left.\right|_{J}}$ to $W^{1, p}(\mathbb{S})$; indeed, given such $\phi_{J}$ and $\phi_{K}$, there is a function $\phi \in W^{1, p}(\mathbb{S})$ that extends the function $\phi_{K}$ defined on $K$. Then $\phi-\phi_{J}=0$ on $\partial K$, and by classical properties of the extension operator (see [26, VI.4.8]) we get that $0_{\left.\right|_{K}} \vee\left(\phi_{J}-\phi\right)_{\left.\right|_{J}} \in W^{1, p}(\mathbb{S})$. Adding $\phi$ we see that $\phi_{K} \vee \phi_{J} \in W^{1, p}(\mathbb{S})$.

In order to approximately recover a function in $H^{p}$ from data available on $K$, we formulate the following Bounded Extremal Problem $\operatorname{BEP}(p)$ :

$\operatorname{BEP}(p)$ : given $p \in(1, \infty), F \in \mathcal{L}_{\nabla}^{p}(K), \Psi \in \mathcal{L}_{\nabla}^{p}(J), M \geq 0$; find $G_{0} \in H^{p}$ such that

$$
\left\|F-G_{0}\right\|_{\mathcal{L}_{\nabla}^{p}(K)}=\min _{\substack{G \in H^{p} \\\|\Psi-G\|_{\mathcal{L}_{\nabla}^{p}(J)} \leq M}}\|F-G\|_{\mathcal{L}_{\nabla}^{p}(K)} .
$$

Note that we conspicuously omit $p=\infty$ from our considerations. Indeed, the solution to the corresponding problem in 2 dimensions uses the Adamjan-Arov-Krein theory [8] which has no analogue in higher dimensions so far.

Proposition $3 A$ solution $G_{0}$ to BEP(p) exists and is unique. Moreover, the constraint is saturated, in the sense that $\left\|\Psi-G_{0}\right\|_{\mathcal{L}_{\nabla}^{p}(J)}=M$ if $F$ is not already the trace, $\sigma$-a.e. on $K$, of an $H^{p}$ function $F_{h}$ with $\left\|\Psi-F_{h}\right\|_{\mathcal{L}_{\nabla}^{p}(J)} \leq M$. 
Proof: We may restrict further the approximating set to those $H^{p}$ functions $G$ such that $\|G\|_{\mathcal{L}_{\nabla}^{p}(K)} \leq$ $2\|F\|_{\mathcal{L}_{\nabla}^{p}(K)}$ for otherwise the zero function is a better candidate anyway. The set of approximating functions is then weakly compact and convex in $H^{p}$, and so is the trace of this set in $\mathcal{L}_{\nabla}^{p}(K)$. The existence and uniqueness now follow because the latter space is a strictly convex Banach space. To check that the constraint is saturated if $\left\|F-G_{0}\right\|_{\mathcal{L}_{\nabla}^{p}(K)} \neq 0$, observe that otherwise there is an $\varepsilon>0$ such that any function $G_{0}+H$ with $H \in H^{p}$ and $\|H\|_{p}<\varepsilon$ also satisfies the constraint. Now, for every $G \in H^{p}$ and $t \in \mathbb{R}$, we get upon differentiating under the integral sign

$$
\begin{aligned}
\left\|F-G_{0}\right\|_{\mathcal{L}_{\nabla}^{p}(K)}^{p} & -\left\|F-G_{0}-t G\right\|_{\mathcal{L}_{\nabla}^{p}(K)}^{p} \\
& =\frac{t p}{2} \int_{K}\left\|F-G_{0}\right\|_{\mathcal{L}_{\nabla}^{p}(K)}^{p-2}\left(F-G_{0}\right) \cdot G d \sigma+o\left(t^{2}\right) .
\end{aligned}
$$

Since the right-hand side must be non-positive as soon as $|t|<\varepsilon /\|G\|_{p}$, we must have that

$$
\int_{K}\left\|F-G_{0}\right\|_{\mathcal{L}_{\nabla}^{p}(K)}^{p-2}\left(F-G_{0}\right) \cdot G d \sigma=0, \quad G \in H^{p} .
$$

Then by using the density result, Lemma 2 we can find a sequence $G_{n}$ in $H^{p}$ that converges to $F-G_{0}$ in $\mathcal{L}_{\nabla}^{p}(K)$, and by virtue of (12) we obtain in the limit $\left\|F-G_{0}\right\|_{\mathcal{L}_{\nabla}^{p}(K)}=0$, a contradiction.

The properties of the solutions to the $\operatorname{BEP}(p)$ are very similar to those observed in two-dimensional situations [2] [. Note first that $\varepsilon=\left\|F-G_{0}\right\|_{\mathcal{L}_{\nabla}^{p}(K)}$ is clearly a decreasing function of $M$, the constraint imposed on $\|\Psi-G\|_{\mathcal{L}_{\nabla}^{p}(J)}$. Given $F$ and $\Psi$ as in $\operatorname{BEP}(p)$ above, there are two situations that must be distinguished.

1. If $F$ is already the trace on $K$ of an $H^{p}$ function $F_{h}$, and we write $M_{0}=\left\|\Psi-F_{h}\right\|_{\mathcal{L}_{\nabla}^{p}(J)}$, then clearly we recover $F_{h}$ exactly as soon as $M \geq M_{0}$, so that $G_{0}=F_{h}$ is a feasible solution to $\operatorname{BEP}(p)$. This is a situation of recovering a function from exact measurements obtained on a set of uniqueness.

2. Otherwise, the behaviour of the solution $G_{0}$ is such that $\varepsilon \rightarrow 0$ as $M \rightarrow \infty$ (this follows essentially from the density result in Theorem 2 and the weak compactness of balls in $H^{p}$ ). In this situation we are approximating noisy data by a feasible model, which is nevertheless an ill-posed problem.

As in any convex optimisation problem, the solution to $\operatorname{BEP}(p)$ can be characterized by a variational equation, which turns out to assume a nicely constructive form in the case $p=2$, where a connection with Toeplitz-type operators arises. When $p \neq 2$, the solution would require the computation of the best approximation projection $L_{\nabla}^{p}(\mathbb{S}) \rightarrow H^{p}$ which is not linear, and for which no closed form is known. Hereafter, we concentrate on the case $p=2$ and we abbreviate $\operatorname{BEP}(2)$ to (BEP).

\subsection{Approximation on the sphere}

Recall the following result from [12, which provides a non-orthogonal generalization of the Hilbertspace techniques of [20].

Proposition 4 [12] Let $A: \mathcal{H} \rightarrow \mathcal{H}_{1}$ and $B: \mathcal{H} \rightarrow \mathcal{H}_{2}$ be Hilbert space operators for which there is a constant $\delta>0$ such that $\|A y\|+\|B y\| \geq \delta\|y\|$ for all $y \in \mathcal{H}$. Take $x_{1} \in \mathcal{H}_{1}$ and $x_{2} \in \mathcal{H}_{2}$. Then the solution to

$$
\left\|A y_{0}-x_{1}\right\|_{\mathcal{H}_{1}}=\inf \left\{\left\|A y-x_{1}\right\|_{\mathcal{H}_{1}}: y \in \mathcal{H},\left\|B y-x_{2}\right\|_{\mathcal{H}_{2}} \leq M\right\}
$$

is given by

$$
\left(A^{*} A+\gamma B^{*} B\right) y_{0}=A^{*} x_{1}+\gamma B^{*} x_{2},
$$

where $\gamma>0$ is the unique constant such that $\left\|B y_{0}-x_{2}\right\|_{\mathcal{H}_{2}}=M$. 
In our situation, it is appropriate to take $\mathcal{H}=H^{2}$, with $A$ and $B$ the restriction mappings to $\mathcal{H}_{1}=\mathcal{L}_{\nabla}^{2}(K)$ and $\mathcal{H}_{2}=\mathcal{L}_{\nabla}^{2}(J)$, respectively.

It is not always convenient to calculate the adjoints, so we can rewrite (13) as

$$
\left\langle A y_{0}-x_{1}, A v\right\rangle_{\mathcal{H}_{1}}+\gamma\left\langle B y_{0}-x_{2}, B v\right\rangle_{\mathcal{H}_{2}}=0 \quad \text { for all } \quad v \in \mathcal{H} .
$$

A Banach space generalization of the above results, valid in $H^{p}$ spaces for $1<p<\infty$, can be found in [13.

The solution to (BEP) may be given by means of a variational equation as follows (see the proposition above, where $A$ is the restriction mapping onto $K$ and $B$ the restriction onto $J$ ). Namely there exists a unique $\gamma \geq 0$ such that

$$
\left\langle G_{0}-F, H\right\rangle_{\mathcal{L}_{\nabla}^{2}(K)}=\gamma\left\langle\Psi-G_{0}, H\right\rangle_{\mathcal{L}_{\nabla}^{2}(J)} \quad \text { for all } \quad H \in H^{2} .
$$

If $F$ is not already the trace on $K$ of an $H^{2}$ function $F_{h}$ such that $\left\|\Psi-F_{h}\right\|_{\mathcal{L}_{\nabla}^{2}(J)} \leq M$ then $\gamma>0$ and $\left\|\Psi-G_{0}(\gamma)\right\|_{\mathcal{L}_{\nabla}^{2}(J)}=M$. Further, it is clear from the variational equation that the case $\gamma \rightarrow 0$ corresponds to $\left\|G_{0}-F\right\|_{\mathcal{L}_{\nabla}^{2}(K)} \rightarrow 0$ and $M \rightarrow \infty$; moreover, $\gamma \rightarrow \infty$ corresponds to $M \rightarrow 0$. Hence, for every $H \in H^{2}$,

$$
\left\langle(I+(\gamma-1) \mathcal{T}) G_{0}, H\right\rangle_{\mathcal{L}_{\nabla}^{2}(\mathbb{S})}=\langle F, H\rangle_{\mathcal{L}_{\nabla}^{2}(K)}+\gamma\langle\Psi, H\rangle_{\mathcal{L}_{\nabla}^{2}(J)}
$$

where $\mathcal{T}=\mathcal{T}_{\chi_{J}}$ is the Toeplitz-like operator on $H^{2}$ (weakly) defined by

$$
\langle\mathcal{T} G, H\rangle_{\mathcal{L}_{\nabla}^{2}(\mathbb{S})}=\langle G, H\rangle_{\mathcal{L}_{\nabla}^{2}(J)}
$$

Now,

$$
\langle\mathcal{T} G, G\rangle_{\mathcal{L}_{\nabla}^{2}(\mathbb{S})}=\|G\|_{\mathcal{L}_{\nabla}^{2}(J)}^{2} \geq 0,
$$

so $\mathcal{T}$ is a positive operator. Moreover, the spectrum of $\mathcal{T}$ is contained in the closed interval $[0,1]$; by analogy with the case of the two-dimensional annulus it is reasonable to conjecture that this spectrum equals the whole of $[0,1]$.

\subsection{The case of the hemisphere in $\mathbb{R}^{3}$}

As an important example of the above situation, take

$$
K=\mathbb{S}_{+}=\left\{\left(x_{1}, x_{2}, x_{3}\right) \in \mathbb{S}, x_{3} \geq 0\right\},
$$

so that $J=\mathbb{S}_{-}=\left\{\left(x_{1}, x_{2}, x_{3}\right) \in \mathbb{S}, x_{3}<0\right\}$, and $\partial K=\partial J=\partial \mathbb{S}_{+}=\left\{\left(x_{1}, x_{2}, x_{3}\right) \in \mathbb{S}, x_{3}=0\right\}$, the unit disk. We derive in this section some explicit formulae to compute the terms involved in the variational equation (14) in this case; more complicated formulae can be derived in other cases: most simply when $K \subset \mathbb{S}$ is a "cap" with circular boundary. The formulae below are those that were used to produce the numerical experiments reported in Section 4

Let $G=\nabla g \in H^{2}$, so we can write from [5] Cor. 5.34]:

$$
g=\sum_{k=1}^{\infty} \gamma_{k} \in L^{2}(\mathbb{S}), \quad \gamma_{k} \in \mathcal{H}_{k}, \quad \sum_{k=1}^{\infty} k^{2}\left\|\gamma_{k}\right\|_{2}^{2}<+\infty,
$$

where the growth condition on the $\left\|\gamma_{k}\right\|_{2}$ is necessary and sufficient for $\nabla g$ to lie in $L^{2}\left(\mathbb{S} ; \mathbb{R}^{3}\right)$ by Euler's identity and the $L^{2}$-boundedness of Riesz transforms. With $p_{m} \in \mathcal{H}_{m}$, we have from the Green formula on $J$, using the intrinsic Laplace-Beltrami operator on the sphere [14, II.7],

$$
\left\langle\nabla_{\mathbb{S}} g, \nabla_{\mathbb{S}} p_{m}\right\rangle_{L^{2}\left(J ; \mathbb{R}^{3}\right)}=\int_{J} \nabla_{\mathbb{S}} g \cdot \nabla_{\mathbb{S}} p_{m}=m(m+1) \int_{J} g p_{m}+\int_{\partial K} g \partial_{x_{3}} p_{m},
$$


hence

$$
\begin{aligned}
\left\langle\nabla g, \nabla p_{m}\right\rangle_{\mathcal{L}_{\nabla}^{2}(J)} & =m(m+1) \int_{J} g p_{m}+m \int_{J} \partial_{n} g p_{m}+\int_{\partial K} g \partial_{x_{3}} p_{m} \\
& =\sum_{k=0}^{\infty}\left[m(m+k+1) \int_{J} \gamma_{k} p_{m}+\int_{\partial K} \gamma_{k} \partial_{x_{3}} p_{m}\right],
\end{aligned}
$$

where $n$ is the unit outer normal vector to $\mathbb{S}(n(\xi)=\xi)$ and $x_{3}=(0,0,1)$ the unit outer normal vector to $\partial J$ which is tangent to $\mathbb{S}$. Also:

$$
\begin{aligned}
\left\langle\nabla g, \nabla p_{m}\right\rangle_{\mathcal{L}_{\nabla}^{2}(K)} & =\int_{K} \nabla g \cdot \nabla p_{m} \\
& =m(m+1) \int_{K} g p_{m}+m \int_{K} \partial_{n} g p_{m}-\int_{\partial K} g \partial_{x_{3}} p_{m} .
\end{aligned}
$$

Adding (17) and (16) (see also [5] Lem. 5.13]), we get by the orthogonality of spherical harmonics of different degrees:

$$
\begin{aligned}
\left\langle\nabla g, \nabla p_{m}\right\rangle_{2} & =\int_{\mathbb{S}} \nabla g \cdot \nabla p_{m} \\
& =m(m+1) \int_{\mathbb{S}} g p_{m}+m \int_{\mathbb{S}} \partial_{n} g p_{m}=m(2 m+1) \int_{\mathbb{S}} \gamma_{m} p_{m}
\end{aligned}
$$

while

$$
\left\langle\nabla_{\mathbb{S}} g, \nabla_{\mathbb{S}} p_{m}\right\rangle_{L^{2}\left(\mathbb{S} ; \mathbb{R}^{3}\right)}=\int_{\mathbb{S}} \nabla_{\mathbb{S}} g \cdot \nabla_{\mathbb{S}} p_{m}=m(m+1) \int_{\mathbb{S}} g p_{m}=m(m+1) \int_{\mathbb{S}} \gamma_{m} p_{m} .
$$

Back to (14) with $g=g_{0}$ and $\nabla g_{0}=G_{0}$, this finally gives:

$$
\begin{aligned}
m(2 m+1) \int_{\mathbb{S}} \gamma_{m} p_{m}+(\gamma-1) \sum_{k=0}^{\infty} & {\left[m(m+k+1) \int_{J} \gamma_{k} p_{m}+\int_{\partial K} \gamma_{k} \partial_{x_{3}} p_{m}\right] } \\
=\int_{K} m\left(f_{0}+(m+1) \phi_{f}\right) p_{m} & +\gamma \int_{J} m\left(\psi_{0}+(m+1) \phi_{\psi}\right) p_{m} \\
& +\int_{\partial K} \phi_{f} \partial_{x_{3}} p_{m}+\gamma \int_{\partial J} \phi_{\psi} \partial_{x_{3}} p_{m},
\end{aligned}
$$

applying (16), (17) to

$$
F=\left(f_{0}, \nabla_{\mathbb{S}} \phi_{f}\right)_{\left.\right|_{K}}, \Psi=\left(\psi_{0}, \nabla_{\mathbb{S}} \phi_{\psi}\right)_{\left.\right|_{J}}
$$

\subsection{Spherical domains}

A similar approximation problem can be stated on the shell $\mathbb{G}$. This may be considered as the analogue of the annulus, for which a similar problem was studied in [19].

Clearly we may formulate a bounded extremal problem corresponding to restrictions to a subset $K \subset \partial \mathbb{G}=\mathbb{S} \cup \rho \mathbb{S}$, for example, $K=\mathbb{S}$ itself. In applications, we will normally be interested only in $K \subseteq \mathbb{S}$, so we limit ourselves to this case.

We first need an analogue to Theorem 2 on the shell.

Lemma 5 If $K \subseteq \mathbb{S}$ is closed then $H^{p}(\mathbb{G})_{\mid K}\left(\right.$ resp. $\left.H_{\mid K}^{c}\right)$ is dense in $\mathcal{L}_{\nabla}^{p}(K)$ (resp. $\left.\mathcal{L}_{\nabla}^{c}(K)\right)$ for $1 \leq p<\infty$. 
Proof: We may suppose that $K=\mathbb{S}$. It is also enough to consider the case of $\mathcal{L}_{\nabla}^{p}(\mathbb{S})$. Now, we saw in the discussion after Remark [3 that any member of $\mathcal{L}_{\nabla}^{p}(\mathbb{S})$ can be approximated uniformly on $\mathbb{S}$ by some polynomial vector field $\left(p, \nabla_{\mathbb{S}} q\right)$, and that any such vector field is the sum of a member of $H^{c}$ and a member of $H_{-}^{c}$, cf. (10).

Let $K$ be a closed subset of $\mathbb{S}$ with a Lipschitz boundary and write $J=\partial \mathbb{G} \backslash K$. The Bounded Extremal Problem now takes the following form:

$\operatorname{BEP}(p, \mathbb{G})$ : Let $1<p<\infty$. Given $F \in \mathcal{L}_{\nabla}^{p}(K), \Psi \in \mathcal{L}_{\nabla}^{p}(J), M \geq 0$; find $G_{0} \in H^{p}(\mathbb{G})$ such that

$$
\left\|F-G_{0}\right\|_{\mathcal{L}_{\nabla}^{p}(K)}=\min _{\substack{G \in H^{p}(\mathbb{G}) \\\|\Psi-G\|_{\mathcal{L}_{\nabla}^{p}(J)} \leq M}}\|F-G\|_{\mathcal{L}_{\nabla}^{p}(K)}
$$

The analogue of Proposition 3 is still valid, namely the solution to the bounded extremal problem is unique. Further, if $F$ is not already the trace on $K$ of an $H^{p}(\mathbb{G})$ function $F_{h}$ such that $\left\|\Psi-F_{h}\right\|_{\mathcal{L}_{\nabla}^{p}(J)} \leq M$ then $\gamma>0$ and $\left\|\Psi-G_{0}(\gamma)\right\|_{\mathcal{L}_{\nabla}^{p}(J)}=M$. These facts are proved exactly as before, using Corollary 1 to reduce to a weakly compact set of approximants and Lemma 5 instead of Lemma 2

Again, the solution is given by a variational equation, and for $p=2$ it assumes the same form as before; namely, there exists a unique $\gamma \geq 0$ such that

$$
\left\langle G_{0}-F, H\right\rangle_{\mathcal{L}_{\nabla}^{2}(K)}=\gamma\left\langle\Psi-G_{0}, H\right\rangle_{\mathcal{L}_{\nabla}^{2}(J)} \quad \text { for all } \quad H \in H^{2}(\mathbb{G}) .
$$

We may again introduce $\mathcal{T}=\mathcal{T}_{\chi_{J}}$, the Toeplitz-like operator on $H^{2}(\mathbb{G})$ (weakly) defined by

$$
\langle\mathcal{T} G, H\rangle_{\mathcal{L}_{\nabla}^{2}(\partial \mathbb{G})}=\langle G, H\rangle_{\mathcal{L}_{\nabla}^{2}(J)} \quad \text { for all } \quad H \in H^{2}(\mathbb{G}) .
$$

In this setting, the variational equation associated to $\operatorname{BEP}(2, \mathbb{G})$ may be written as:

$$
\left\langle(I+(\gamma-1) \mathcal{T}) G_{0}, H\right\rangle_{\mathcal{L}_{\nabla}^{2}(\partial \mathbb{G})}=\langle F, H\rangle_{\mathcal{L}_{\nabla}^{2}(K)}+\gamma\langle\Psi, H\rangle_{\mathcal{L}_{\nabla}^{2}(J)}
$$

for all $H \in H^{2}(\mathbb{G})$, and in the particular case where $K=\mathbb{S}, J=\rho \mathbb{S}$, we get

$$
\left\langle G_{0}, H\right\rangle_{L^{2}\left(\mathbb{S} ; \mathbb{R}^{n}\right)}+\gamma\left\langle G_{0}, H\right\rangle_{L^{2}\left(\rho \mathbb{S} ; \mathbb{R}^{n}\right)}=\langle F, H\rangle_{L^{2}\left(\mathbb{S} ; \mathbb{R}^{n}\right)}+\gamma\langle\Psi, H\rangle_{L^{2}\left(\rho \mathbb{S} ; \mathbb{R}^{n}\right)} .
$$

Now every harmonic function on $\mathbb{G}$ decomposes as $g(r, \sigma)=\sum_{k=-\infty}^{\infty} r^{k} S_{k}(\sigma)$, terms of index $k \geq 0$ being harmonic on $\mathbb{B}$ and terms of index $k<0$ being harmonic on $\mathbb{R}^{n} \backslash \rho \overline{\mathbb{B}}$ (see [5]) or:

$$
\begin{aligned}
g(X)=g(r, \sigma) & =\sum_{k=0}^{\infty} \gamma_{k}(X)+\mathcal{K}\left[q_{k}\right](X)=\sum_{k=0}^{\infty} \gamma_{k}(X)+\frac{q_{k}(X)}{|X|^{2 k+n-2}} \\
& =\sum_{k=0}^{\infty} r^{k} \gamma_{k}(\sigma)+r^{-(k+n-2)} q_{k}(\sigma), \quad \gamma_{k}, q_{k} \in \mathcal{H}_{k} .
\end{aligned}
$$

By Proposition 2 the condition for $\nabla g$ to lie in $H^{2}(\mathbb{G})$ is that

$$
\sum_{k=0}^{\infty} k^{2}\left\|\gamma_{k}\right\|_{2}^{2}<+\infty \quad \text { and } \quad \sum_{k=0}^{\infty}(k / \rho)^{2}\left\|q_{k}\right\|_{2}^{2}<+\infty
$$

In particular when $n=3$, we obtain in place of (18), with $g_{0}$ given by (20):

$$
m(2 m+1)\left[1+\gamma \rho^{2 m}\right] \int_{\mathbb{S}} \gamma_{m} p_{m}=(m+1)(2 m+1)\left[1+\gamma \rho^{-2(m+1)}\right] \int_{\mathbb{S}} q_{m} p_{m}
$$




$$
=\int_{\mathbb{S}} m\left(f_{0}+(m+1) \phi_{f}\right) p_{m}+\gamma \int_{\rho \mathbb{S}} m\left(\psi_{0}+(m+1) \phi_{\psi}\right) p_{m},
$$

for prescribed functions $F, \Psi$ as (19) with $K=\mathbb{S}$ and $J=\rho \mathbb{S}$ :

$$
F=\left(f_{0}, \nabla_{\mathbb{S}} \phi_{f}\right)_{\left.\right|_{\mathbb{S}}}, \Psi=\left(\psi_{0}, \nabla_{\mathbb{S}} \phi_{\psi}\right)_{\left.\right|_{\rho \mathbb{S}}} .
$$

So, for $K=\mathbb{S}$, we see that, as in the two-dimensional situation of the annulus [19, 25], the Toeplitz operator $\mathcal{T}$ is already diagonal with respect to the orthonormal basis of spherical harmonics, which makes the numerical calculation of $G_{0}$ particularly easy to implement.

\section{Numerical computations}

Let $g$ be a function harmonic in a domain $\mathcal{D} \subset \mathbb{R}^{3}$. Assume that we are given measurements $g$ and $\partial_{n} g$ on $K \subset \partial \mathcal{D}$, from which we wish to reconstruct the function $g$. We will consider the following situations, as in Sections 3.2 and 3.3

(i) $\mathcal{D}=\mathbb{B}, K=\mathbb{S}_{+} \subset \mathbb{S}, J=\mathbb{S}_{-} \subset \mathbb{S}$;

(ii) $\mathcal{D}=\mathbb{G}, K=\mathbb{S} \subset \partial \mathbb{G}, J=\rho \mathbb{S} \subset \partial \mathbb{G}$.

Assume also, in (19), the data to be given on $K$ by the trace there of their decompositions into spherical harmonics:

$$
\phi_{f}=\left(\sum_{k=0}^{\infty} \varphi_{k}\right)_{\left.\right|_{K}}, \quad f_{0}=\left(\sum_{k=0}^{\infty} \delta_{k}\right)_{\left.\right|_{K}} \text { while } \psi_{0}=0, \quad \phi_{\psi}=0 \text { on } J .
$$

\subsection{Situation $(i)$}

Looking at the decomposition (15) of $g_{0}$ where $\nabla g_{0}$ solves $\operatorname{BEP}(2, \mathbb{S})$ in $\mathbb{R}^{3}$, that is, $g_{0}$ solves (18) for each $p_{m} \in \mathcal{H}_{m}$ and all $m$, with data given on $K=\mathbb{S}_{+}$and $J=\mathbb{S}_{-}$as traces of functions in $L^{2}(\mathbb{S})$ by (22), we get:

$$
\begin{gathered}
m(2 m+1) \int_{\mathbb{S}} \gamma_{m} p_{m}+(\gamma-1) \sum_{k=0}^{\infty}\left[m(m+k+1) \int_{\mathbb{S}_{+}} \gamma_{k} p_{m}+\int_{\partial \mathbb{S}_{+}} \gamma_{k} \partial_{x_{3}} p_{m}\right] \\
=\int_{\mathbb{S}_{+}} m\left(f_{0}+(m+1) \phi_{f}\right) p_{m}+\int_{\partial \mathbb{S}_{+}} \phi_{f} \partial_{x_{3}} p_{m} \\
=\sum_{k=0}^{\infty}\left[\int_{\mathbb{S}_{+}} m\left(\delta_{k}+(m+1) \varphi_{k}\right) p_{m}+\int_{\partial \mathbb{S}_{+}} \varphi_{k} \partial_{x_{3}} p_{m}\right] .
\end{gathered}
$$

We express these quantities in terms of the basis of spherical harmonics on $\mathbb{S}$ :

$$
\gamma_{k}(X)=\gamma_{k}(r, \sigma)=r^{k} \sum_{i=-k}^{k} \alpha_{k}^{i} Y_{k}^{i}(\sigma),
$$

where $Y_{m}^{i}$ are the $2 m+1$ Legendre polynomials of degree $m$, [5] 15], which are pairwise orthogonal in $L^{2}(\mathbb{S})$. Choosing

$$
p_{m}(X)=r^{m} Y_{m}^{j}(\sigma),
$$

for some $j \in\{-m, \cdots, m\}$, we can rewrite the terms involving the unknown quantities $\left(\alpha_{k}^{i}\right)$ in the above equation as:

$$
\begin{aligned}
m(2 m+1) \alpha_{m}^{j}+(\gamma-1) \sum_{k=0}^{\infty} \sum_{i=-k}^{k} \quad & \alpha_{k}^{i}\left[m(m+k+1) \int_{\mathbb{S}_{+}} Y_{k}^{i}(\sigma) Y_{m}^{j}(\sigma) d \sigma\right. \\
& \left.+\int_{\partial \mathbb{S}_{+}} Y_{k}^{i}(\tau) \partial_{x_{3}}\left(Y_{m}^{j}\right)(\tau) d \tau\right]
\end{aligned}
$$

$\mathrm{RR} \mathrm{n}^{\circ} 6504$ 
and analogously for the right-hand side with the given data on $\mathbb{S}_{+}$:

$$
\phi_{f}(\sigma)=\sum_{k=0}^{\infty} \sum_{i=-k}^{k} \mu_{k}^{i} Y_{k}^{i}(\sigma), f_{0}(\sigma)=\sum_{k=0}^{\infty} \sum_{i=-k}^{k} \nu_{k}^{i} Y_{k}^{i}(\sigma)
$$

which can be written as:

$$
\begin{gathered}
\sum_{k=0}^{\infty} \sum_{i=-k}^{k}\left[m \nu_{k}^{i} \int_{\mathbb{S}_{+}} Y_{k}^{i}(\sigma) Y_{m}^{j}(\sigma) d \sigma\right. \\
\left.+\mu_{k}^{i}\left(m(m+1) \int_{\mathbb{S}_{+}} Y_{k}^{i}(\sigma) Y_{m}^{j}(\sigma) d \sigma+\int_{\partial \mathbb{S}_{+}} Y_{k}^{i}(\tau) \partial_{x_{3}}\left(Y_{m}^{j}\right)(\tau) d \tau\right)\right] .
\end{gathered}
$$

The computations are then performed by truncating the decomposition of $g, f_{0}$ and $\phi_{f}$ on the basis, and by solving the obtained system of linear equations.

Observe that though the following computations already give a good illustration of the method, a full analysis of the numerical behaviour of the approximation scheme is still to be made, especially for data arising in applications. Some simplifications do arise in the case of $\mathbb{S}_{+}$, due to the symmetry properties of the functions $\left(Y_{k}^{i}\right)$, even though they do not form an orthogonal basis when restricted to the hemisphere.

Assume now that we are given on $K=\mathbb{S}_{+}$the trace and the trace of the normal derivative for the function (harmonic in $\mathbb{B}$ ):

$$
g(X)=\sum_{i=1}^{3} \frac{|X|}{\left.\left|X-M_{i}\right| X\right|^{2} \mid}, \phi_{f}=g_{\left.\right|_{\mathbb{S}_{+}}}, f_{0}=\left(\partial_{n} g\right)_{\left.\right|_{\mathbb{s}_{+}}},
$$

where

$$
M_{1}=\left(\begin{array}{l}
0.1 \\
0.2 \\
0.2
\end{array}\right), M_{2}=\left(\begin{array}{l}
0.2 \\
0.2 \\
0.25
\end{array}\right), M_{3}=\left(\begin{array}{l}
0.2 \\
0.25 \\
0.3
\end{array}\right) .
$$

Here as $\gamma \rightarrow 0$, we obtain exact recovery of $g$ because $\nabla g \in H^{2}$. However, $g_{\left.\right|_{K}}$ also coincides with the trace on $K\left(F=\nabla g_{\left.\right|_{K}}\right)$ of a function with singularities $M_{i}$ in $\mathbb{B}$, namely

$$
\sum_{i=1}^{3} \frac{1}{\left|X-M_{i}\right|}
$$

Figure $\prod$ shows a comparison between the function $g_{0}$ on $\mathbb{S}_{+}$and the approximations produced as $\gamma \rightarrow 0$. The present computations were performed using the above procedure in Matlab. It is seen that for sufficiently small $\gamma$ we obtain very good recovery on $\mathbb{S}_{+}$.

\subsection{Situation $(i i)$}

In the shell, taking the decomposition (20) of $g_{0}$ where $\nabla g_{0}$ solves $\operatorname{BEP}(2, \mathbb{G})$ in $\mathbb{R}^{3}$ with data on $\mathbb{S}$, we get from (21) that:

$$
\begin{gathered}
m(2 m+1)\left[1+\gamma \rho^{2 m}\right] \int_{\mathbb{S}} \gamma_{m} p_{m} \\
=(m+1)(2 m+1)\left[1+\gamma \rho^{-2(m+1)}\right] \int_{\mathbb{S}} q_{m} p_{m} \\
=\int_{\mathbb{S}} m\left(f_{0}+(m+1) \phi_{f}\right) p_{m} .
\end{gathered}
$$

In this diagonal case, we simply get from (22) with $K=\mathbb{S}$ and $J=\rho \mathbb{S}$ that, for every $m \geq 0$ :

$$
\gamma_{m}=\frac{\delta_{m}+(m+1) \varphi_{m}}{(2 m+1)\left[1+\gamma \rho^{2 m}\right]}, q_{m}=\frac{m\left(\delta_{m}+(m+1) \varphi_{m}\right)}{(m+1)(2 m+1)\left[1+\gamma \rho^{-2(m+1)}\right]} .
$$



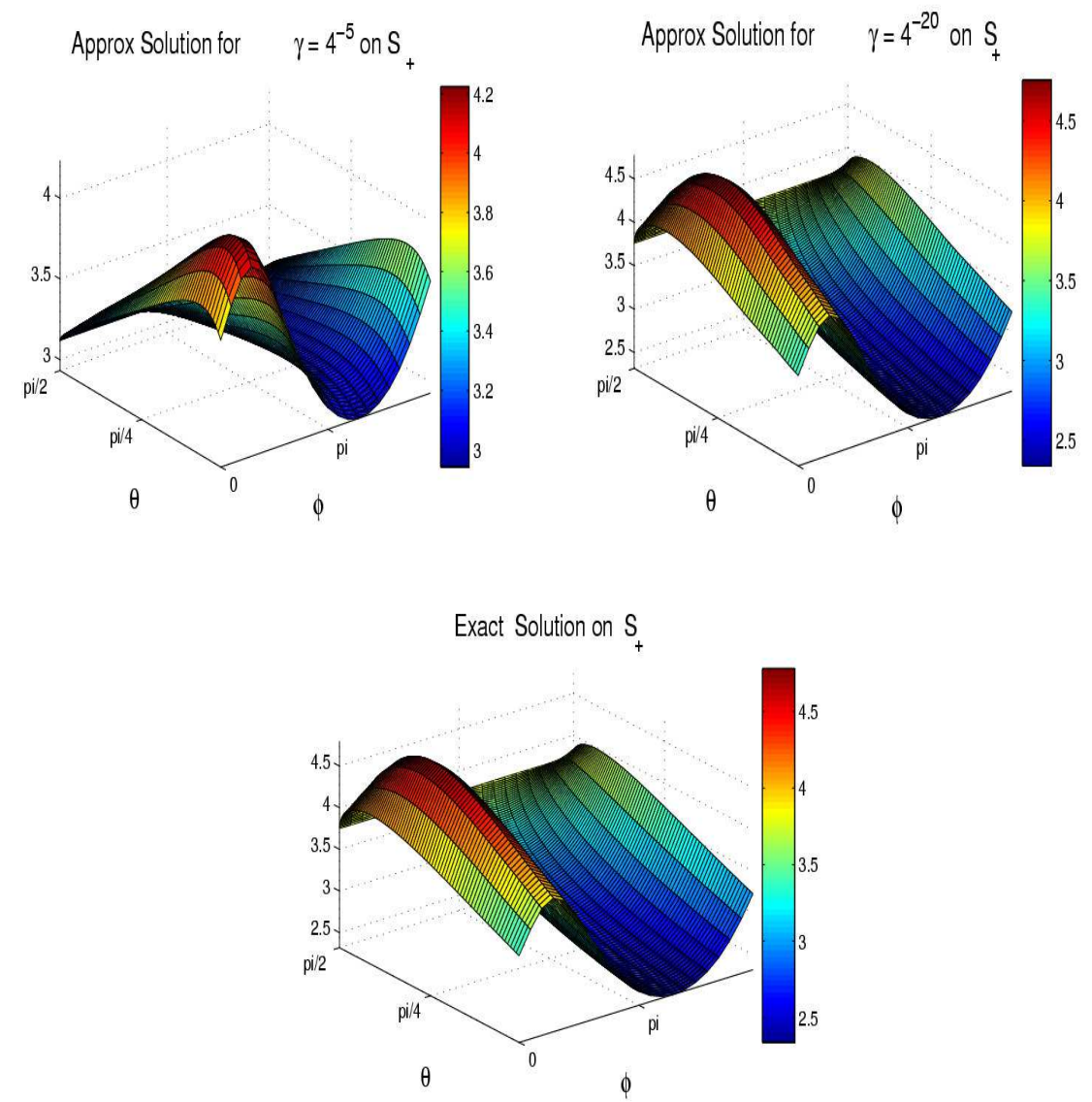

Figure 1: Solution $g_{0}$ compared to the function $g$ on $K=\mathbb{S}_{+}$ 
Observe that whenever $F=\nabla g_{\left.\right|_{\mathbb{S}}} \in H^{2}(\mathbb{G})_{\left.\right|_{\mathbb{S}}}$ :

$$
\left.g\right|_{\mathbb{S}}=\phi_{f}=\sum_{k=0}^{\infty} \varphi_{k},\left.\quad \partial_{n} g\right|_{\mathbb{S}}=f_{0}=\sum_{k=0}^{\infty} \delta_{k}
$$

with $g$ as in (20), we directly obtain the relations (corresponding to the case where $\gamma=0$ in (24)):

$$
\begin{aligned}
\gamma_{k}+q_{k} & =\varphi_{k}, \\
k \gamma_{k}-(k+1) q_{k} & =\delta_{k} .
\end{aligned}
$$

which give:

$$
\begin{aligned}
& \gamma_{k}=\frac{(k+1) \varphi_{k}+\delta_{k}}{2 k+1} \\
& q_{k}=\frac{k \varphi_{k}-\delta_{k}}{2 k+1} .
\end{aligned}
$$

We assume that we are given on $K=\mathbb{S}$ the trace and the trace of the normal derivative of the function (harmonic in $\mathbb{G}=\mathbb{B} \backslash 0.9 \overline{\mathbb{B}}$ ):

$$
g(X)=\sum_{i=1}^{3} \frac{1}{\left|X-M_{i}\right|}
$$

where the $M_{i}$ are the same as in Situation $(i)$.

Figure 2 shows a comparison between the function $g_{0}$ on $\mathbb{S}$ and the approximations produced as $\gamma \rightarrow 0$. It is seen once more that, for sufficiently small $\gamma$, we obtain very good recovery on $\mathbb{S}$.

In this situation, the computations using a basis of spherical harmonics proceed as follows:

$$
\gamma_{k}(X)=\gamma_{k}(r, \sigma)=r^{k} \sum_{i=-k}^{k} \alpha_{k}^{i} Y_{k}^{i}(\sigma), \quad q_{k}(X)=q_{k}(r, \sigma)=r^{k} \sum_{i=-k}^{k} \beta_{k}^{i} Y_{k}^{i}(\sigma) .
$$

or

$$
g(r, \sigma)=\sum_{k=0}^{\infty}\left(r^{k} \sum_{i=-k}^{k} \alpha_{k}^{i} Y_{k}^{i}(\sigma)+r^{-(k+1)} \sum_{i=-k}^{k} \beta_{k}^{i} Y_{k}^{i}(\sigma)\right)
$$

while $\phi_{f}$ and $f_{0}$ are defined on $\mathbb{S}$ by (23). This gives:

$$
\begin{aligned}
\alpha_{k}^{i}+\beta_{k}^{i} & =\mu_{k}^{i}, \\
k \alpha_{k}^{i}-(k+1) \beta_{k}^{i} & =\nu_{k}^{i} .
\end{aligned}
$$

This gives the unknown coefficients $\alpha_{k}^{i}$ and $\beta_{k}^{i}$ by:

$$
\begin{aligned}
\alpha_{k}^{i} & =\frac{k+1}{2 k+1} \mu_{k}^{i}+\frac{1}{2 k+1} \nu_{k}^{i}, \\
\beta_{k}^{i} & =\frac{k}{2 k+1} \mu_{k}^{i}-\frac{1}{2 k+1} \nu_{k}^{i} .
\end{aligned}
$$

We have harmonic extensions of the spherical harmonics, namely $\left(r^{k} Y_{k}^{i}\right)_{i=-k}^{k}$, the homogeneous harmonic polynomials of degree $k$ defined inside $\mathbb{B}$, and $\left(r^{-(k+1)} Y_{k}^{i}\right)_{i=-k}^{k}$, the homogeneous "antiharmonic" polynomials defined on the complement of $\mathbb{B}$. Also,

$$
\partial_{n}\left(r^{k} Y_{k}^{i}\right)_{\left.\right|_{\mathbb{S}}}=k Y_{k}^{i}, \quad \partial_{n}\left(r^{-(k+1)} Y_{k}^{i}\right)_{\left.\right|_{\mathbb{S}}}=-(k+1) Y_{k}^{i} .
$$



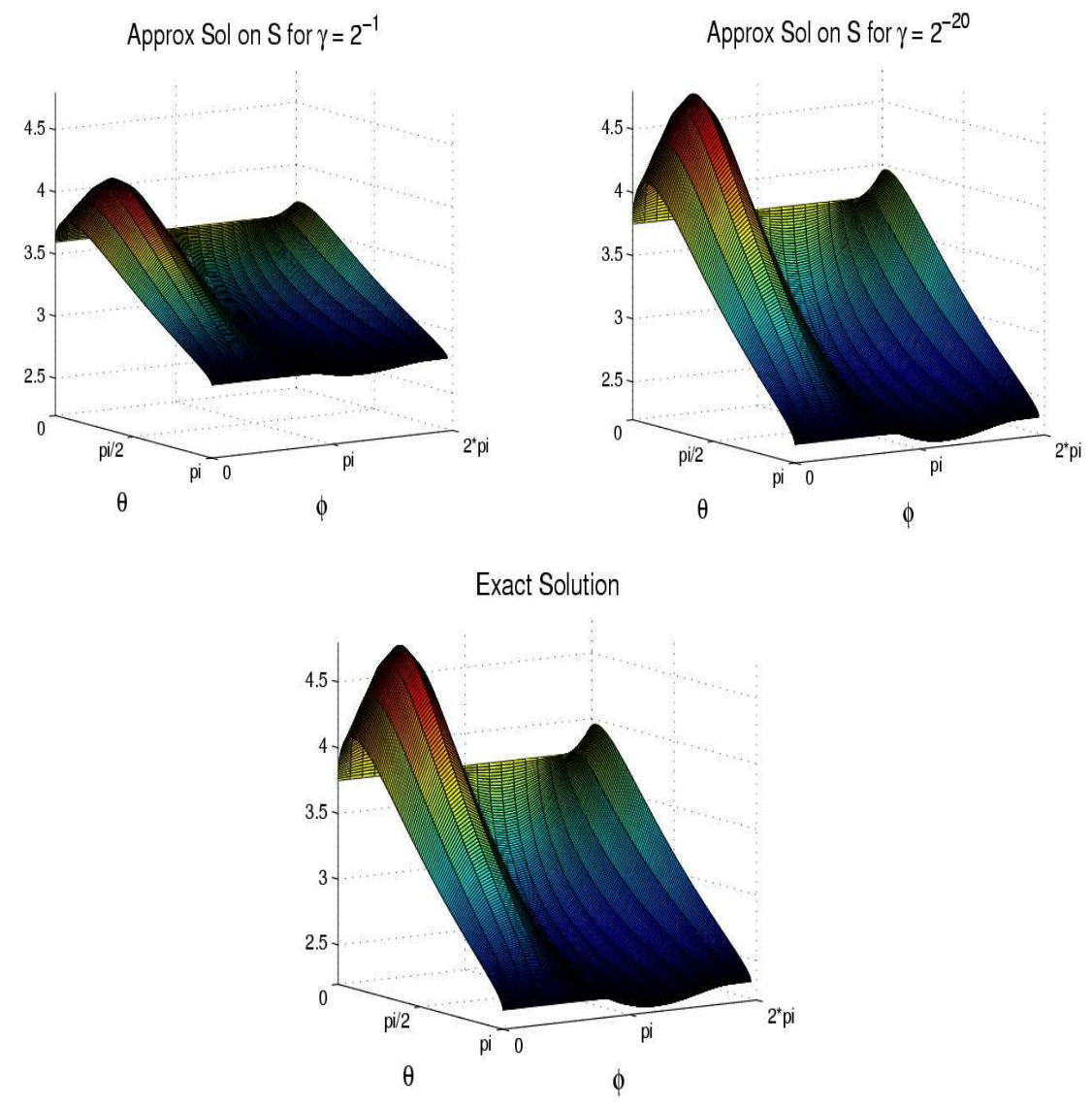

Figure 2: Solution $g_{0}$ compared to the function $g$ on $K=\mathbb{S}$ 


\section{Conclusions}

Although we have formulated our approximation problems in terms of spheres and spherical shells, being driven by concrete applications, it is clear that analogous problems can be considered on a half-space such as $\mathbb{R}_{+}^{n}=\left\{x=\left(x_{1}, \ldots, x_{n}\right) \in \mathbb{R}^{n}: x_{1}>0\right\}$ (indeed much of the work of Stein and Weiss 26, 27, 28] was first expressed in this context).

Further work in this direction will focus on a more detailed numerical analysis of the approximation scheme introduced in this paper. Further extensions to $\mathcal{D}=\mathbb{G}, K=\mathbb{S}_{+} \subset \partial \mathbb{S} \subset \partial \mathbb{G}$ are

also of scientific importance. The main technical difficulties, as in Section 4.1 above, lie in the calculation of the decomposition of the solution into spherical harmonics.

\section{Acknowledgments}

The second author wishes to thank P. Jaming and S. Grellier for fruitful discussions. The third and fourth authors are grateful to the EPSRC for financial support under Grant EP/F020341/1.

\section{References}

\section{References}

[1] R. A. Adams. Sobolev spaces, Academic Press, Orlando, 1975.

[2] D. Alpay, L. Baratchart and J. Leblond. Some extremal problems linked with identification from partial frequency data. Analysis and optimization of systems: state and frequency domain approaches for infinite-dimensional systems (Sophia-Antipolis, 1992), 563-573, Lecture Notes in Control and Inform. Sci., 185, Springer, Berlin, 1993.

[3] N. Arcozzi. Riesz transforms on compact Lie groups, spheres and Gauss space. Ark. Mat. 36 (1998), no. 2, 201-231.

[4] N. Arcozzi and X. Li. Riesz transforms on spheres. Math. Res. Lett. 4 (1997), no. 2-3, 401-412.

[5] S. Axler, P. Bourdon and W. Ramey. Harmonic Function Theory. Springer, 2nd ed., 2001.

[6] L. Baratchart and J. Leblond. Hardy approximation to $L^{p}$ functions on subsets of the circle with $1 \leq p<\infty$. Constr. Approx. 14 (1998), no. 1, 41-56.

[7] L. Baratchart, J. Leblond and J.-P. Marmorat. Inverse source problem in a 3D ball from best meromorphic approximation on 2D slices. Elec. Trans. Num. Anal., 25 (2006), 41-53.

[8] L. Baratchart, J. Leblond and J.R. Partington. Hardy approximation to $L^{\infty}$ functions on subsets of the circle. Constr. Approx. 12 (1996), 423-436.

[9] L. Bers, F. John and M. Schechter. Partial differential equations. Lectures in Applied Mathematics, 3A. American Mathematical Society, Providence, R.I., 1979.

[10] J. Bourgain and T. Wolff. A remark on gradients of harmonic functions in dimension $d \geq 3$. Colloq. Math., 60/61 (1990), 253-260.

[11] I. Chalendar, J. Leblond and J.R. Partington. Approximation problems in some holomorphic spaces, with applications. Systems, approximation, singular integral operators, and related topics (Bordeaux, 2000), 143-168, Oper. Theory Adv. Appl., 129, Birkhäuser, Basel, 2001.

[12] I. Chalendar and J.R. Partington. Constrained approximation and invariant subspaces. J. Math. Anal. Appl. 280 (2003), no. 1, 176-187. 
[13] I. Chalendar, J.R. Partington and M. Smith. Approximation in reflexive Banach spaces and applications to the invariant subspace problem. Proc. Amer. Math. Soc. 132 (2004), no. 4, 1133-1142.

[14] R. Dautray and J.-L. Lions. Analyse Mathématique et Calcul Numérique, volume 2. SpringerVerlag, 1990.

[15] R. Dautray and J.-L. Lions. Mathematical analysis and numerical methods for science and technology, volume 1. Springer-Verlag, 1990.

[16] M. Hämäläinen, R. Hari, J. Ilmoniemi, J. Knuutila and O.V. Lounasmaa. Magnetoencephalography - theory, instrumentation, and applications to noninvasive studies of the working human brain. Reviews of Modern Physics, 65 (1993), no. 2, 413-497.

[17] V. Isakov. Inverse source problems, Mathematical Surveys and Monographs, vol. 34, American Mathematical Society, Providence, RI, 1990.

[18] J. Kybic, M. Clerc, T. Abboud, O. Faugeras, R. Keriven and T. Papadopoulo. A common formalism for the integral formulations of the forward EEG problem, IEEE Transactions on Medical Imaging, 24 (2005), 12-28.

[19] J. Leblond, M. Mahjoub and J.R. Partington. Analytic extensions and Cauchy-type inverse problems on annular domains: stability results. J. Inverse Ill-Posed Probl. 14 (2006), no. 2, 189-204.

[20] J. Leblond and J.R. Partington. Constrained approximation and interpolation in Hilbert function spaces. J. Math. Anal. Appl. 234 (1999), no. 2, 500-513.

[21] J.-L. Lions and E. Magenes. Problèmes aux limites non homogènes et applications. vol. 1, Dunod, Paris, 1971.

[22] G. G. Lorentz. Approximation of functions. Chelsea, New York, 1986.

[23] J.R. Partington. Interpolation, identification, and sampling. London Mathematical Society Monographs. New Series, 17. The Clarendon Press, Oxford University Press, New York, 1997.

[24] J. Rauch. Partial differential equations. Graduate Texts in Mathematics, 128. Springer-Verlag, New York, 1991.

[25] M. Smith. The spectral theory of Toeplitz operators applied to approximation problems in Hilbert spaces. Constr. Approx. 22 (2005), no. 1, 47-65.

[26] E.M. Stein. Singular integrals and differentiability properties of functions. Princeton Mathematical Series, No. 30 Princeton University Press, Princeton, N.J. 1970.

[27] E.M. Stein. Harmonic analysis: real-variable methods, orthogonality, and oscillatory integrals. Princeton Mathematical Series, 43. Monographs in Harmonic Analysis, III. Princeton University Press, Princeton, NJ, 1993.

[28] E.M. Stein and G. Weiss. Introduction to Fourier analysis on euclidean spaces. Princeton Univ. Press, 1971.

[29] A. Torchinsky. Real-variable Methods in Harmonic Analysis. Academic Press, San Diego, 1986.

[30] W. Wang. A remark on gradients of harmonic functions. Rev. Mat. Iberoamer., 11 (1995), no. $2,227-245$.

[31] T. Wolff. Counterexamples with harmonic gradients in $\mathbf{R}^{3}$. Essays on Fourier analysis in honor of Elias M. Stein (Princeton, NJ, 1991), 321-384, Math. Ser., 42, Princeton Univ. Press, 1995. 
[32] W. P. Ziemer. Weakly Differentiable Functions. Graduate Texts in Mathematics, 120. Springer-Verlag, New York, 1989. 


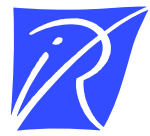

Centre de recherche INRIA Sophia Antipolis - Méditerranée 2004, route des Lucioles - BP 93 - 06902 Sophia Antipolis Cedex (France)

Centre de recherche INRIA Bordeaux - Sud Ouest : Domaine Universitaire - 351, cours de la Libération - 33405 Talence Cedex Centre de recherche INRIA Grenoble - Rhône-Alpes : 655, avenue de l'Europe - 38334 Montbonnot Saint-Ismier Centre de recherche INRIA Lille - Nord Europe : Parc Scientifique de la Haute Borne - 40, avenue Halley - 59650 Villeneuve d'Ascq Centre de recherche INRIA Nancy - Grand Est : LORIA, Technopôle de Nancy-Brabois - Campus scientifique 615, rue du Jardin Botanique - BP 101 - 54602 Villers-lès-Nancy Cedex

Centre de recherche INRIA Paris - Rocquencourt : Domaine de Voluceau - Rocquencourt - BP 105 - 78153 Le Chesnay Cedex

Centre de recherche INRIA Rennes - Bretagne Atlantique : IRISA, Campus universitaire de Beaulieu - 35042 Rennes Cedex Centre de recherche INRIA Saclay - Île-de-France : Parc Orsay Université - ZAC des Vignes : 4, rue Jacques Monod - 91893 Orsay Cedex 